Pacific Journal of Mathematics

HILBERT TRANSFORMS, AND A PROBLEM IN SCATTERING 


\title{
HILBERT TRANSFORMS, AND A PROBLEM IN SCATTERING THEORY
}

\author{
M. J. WESTWATER
}

Let $\mathfrak{S}$ be a separable infinite dimensional complex Hilbert space, $B(\mathfrak{S})$ the set of bounded linear operators on S. Consider a holomorphic map $z \rightarrow K(z)$ from a complex neighborhood of some interval of the real axis to $B(\mathfrak{S})$, such that for $z$ real $K(z)$ is hermitian. These conditions are satisfied by $K(z)=H-z 1$, with $H$ hermitian. In this special case $K(z)$ has a bounded inverse $S(z)$ (the resolvent of $H$ ), for $z$ not on the real axis, and $S(z)$ can be represented as the Hilbert transform of a measure whose values are bounded positive operators (the spectral measure of $H$ ); for $z$ on the real axis $K(z)$ has a (generally unbounded) inverse for $z$ not in the point spectrum of $H$; closely related to the spectral representation of $S(z)$ is an approximation theorem which asserts roughly that for most real values of $z,[K(z)]^{-1}$ can be approximated by operators of finite rank obtained by taking the orthogonal projector $P$ onto a finite dimensional subspace $D$ and inverting $P K(z) P$ on $D$. The object of this paper is to give conditions on $K(z)$ sufficient to imply the conlusions just noted in the special case $K(z)=H-z 1$.

The main theorems are Theorem 1 in $\S 1$, and Theorem 4 in $\S 4$; each has two parts - a representation for $[K(z)]^{-1}$ for $z$ complex, and an approximation theorem for $[K(z)]^{-1}$ for $z$ real. Theorem 4 is used in $\S 5$ to prove a convergence theorem (Theorem 5) for the Kohn variational method in quantum mechanical potential scattering (the relevant terms are defined in that section). This application motivated the writing of the paper.

1. The representation theorem. Throughout this paper we will be working in a separable complex Hilbert space $\mathfrak{S}_{\text {. }} \mu$ will denote Lebesgue measure on the real line $\boldsymbol{R}$. We will need to consider positive operator valued measures on $\boldsymbol{R}$. Such a measure is a map $\boldsymbol{\nu}$ from Borel subsets $B$ of $\boldsymbol{R}$ to positive bounded operators on $\mathfrak{K}$ such that for any $f, g \in \mathfrak{S}$ the map $\nu_{f, g}: B \rightarrow(f, \nu(B) g)$ is a complex measure on $\boldsymbol{R}$. If $\left\{\boldsymbol{\nu}_{n}\right\}$ is a sequence of Borel measures on $\boldsymbol{R}$, with the total variation of $\nu_{n}$ bounded in $n$, the convergence $\nu_{n} \rightarrow \nu$ of $\nu_{n}$ to a measure $\nu$ will always mean weak* convergence (also called vague convergence).

We begin by stating our main result in its simplest form. This will be further generalized in $\S 4$. 
THEOREM 1. Let $\{K(z)\}$ be a family of bounded operators on $\mathfrak{S}$, defined and holomorphic in $z$, for $z$ in a complex neighborhood of some finite open interval $I$ of the real axis. Suppose that

(a) $K(z)$ is hermitian for $z \in I$

(b) $K^{\prime}(z)=1+C(z)$, with $C(z)$ compact, for $z \in U$

(c) for some $z \in U-I, K(z)$ has a bounded inverse.

Then there exists a discrete set $E \subset I$ such that, for each open interval $J$ whose closure is contained in $I-E, K(z)$ has a bounded inverse $S(z)$ for $z \in N_{J}-J$ (where $N_{J}$ is a certain complex neighborhood of $J)$. In $N_{J}-J, S(z)$ admits a representation

$$
S(z)=\int_{J} \frac{d \nu(t)}{z-t}+R_{J}(z)
$$

where $\nu$ is a positive operator valued measure on $J$, and $R_{J}(z)$ is holomorphic in $N_{J}$.

Let $\left\{D_{N}\right\}$ be an increasing sequence of finite dimensional subspaces of $\mathfrak{K}$, whose union $D$ is dense in $\mathfrak{K}$. Denote by $P_{N}$ the orthogonal projection onto $D_{N}$. For sufficiently large $N$ the restriction of $P_{N} K(z) P_{N}$ to $D_{N}$ is invertible (as an operator on $D_{N}$ ) for all but a discrete set of $z \in I$ (dependent on $N)$; when this inverse is defined we extend it to an operator $T_{N}(z)$ on $\mathfrak{S}_{\text {by }}$ betting $T_{N}(z)=0$ on the orthogonal complement of $D_{N}$. Then as $N \rightarrow \infty$

$$
T_{N}(z) K(z) \longrightarrow 1
$$

weakly in measure $(\mu)$ on $I$, i.e., for all $f, g \in \mathfrak{S}$

$$
\left(f, T_{N}(z) K(z) g\right) \longrightarrow(f, g)
$$

in measure on $I$.

REMARK. For $C(z)=0, K(z)$ in Theorem 1 is of the form $-H+z 1$ with $H$ hermitian, and $S(z)$ is the resolvent of $H$. There is no exceptional set $E$, and (1.1) is the representation of $S(z)$ given by the spectral theorem.

Proof of Theorem 1. The proof of Theorem 1 follows from:

(i) The special case where $\|C(z)\| \leqslant \sigma<1$

(ii) A perturbation result involving preservation of the validity of the conclusions of Theorem 1 under an additive perturbation of $C(z)$ by a constant rank 1 operator. The results we obtain in these special cases are somewhat stronger than are necessary for the proof of Theorem 1. We state them as Theorems 2 and 3.

THEOREM 2. Let $\{V(z)\}$ be a family of bounded operators on $\mathfrak{S}$, 
defined and holomorphic in $z$, for $z$ in a complex neighborhood $U$ of some finite open interval $I$ of the real axis. Suppose that the intersection of $U$ with any line $\operatorname{Re} z=\alpha$ is either empty or connected. Let $H$ be a self-adjoint operator and $D$ a core for $H$. Suppose that

(a) $K(z)=-H-V(z)+z 1$

(b) $\left\|V^{\prime}(z)\right\| \leqslant \sigma<1 \quad$ for $z \in U$.

Then $K(z)$ has a bounded inverse $S(z)$ for $z \in U-I$. For each open interval $J$ whose closure is contained in $I, S(z)$ admits for $z \in U-I$ a representation

$$
S(z)=\int_{J} \frac{d \nu(t)}{z-t}+R_{J}(z)
$$

where $\nu$ is a positive operator valued measure on $J$, and $R_{J}(z)$ is holomorphic in $U-(I-J)$. If $D$ is the union of an increasing sequence of finite dimensional subspaces $D_{N}$ of $\mathfrak{K}_{\mathcal{C}}$, and $T_{N}(z)$ is defined as in Theorem 1

$$
\left(f, T_{N}(z) K(z) g\right) \longrightarrow(f, g)
$$

as $N \rightarrow \infty$ in measure $(\mu)$ on $I$, for $f \in \mathfrak{F}, g \in$ domain of $H$.

TheOREM 3. Let $\{S(z)\}$ be family of bounded operators on $\mathfrak{S}$ defined and holomorphic in $z$, for all $z$ with $\operatorname{Im} z \neq 0$ in a connected open set $U$ containing an open subset of $I-E$ of the real axis. Here $I$ is a finite open interval, and $E$ a discrete subset of $I$. Suppose that, for any open interval $J$ whose closure is contained in $I-E$, there exists a complex neighborhood $N_{J}$ of $J$ such that, for $z \in N_{J}-J, S(z)$ admits a representation (1.1). Let $K$ be a symmetric rank 1 operator.

Then either $1-z$ trace $(K S(z))=0, z \in U, \operatorname{Im} z \neq 0$ or with $a$ suitably defined discrete set $\widetilde{E} \supset E$ replacing $E$, and a connected open set $\widetilde{U} \supset I-\widetilde{E}$ replacing $U$, the family of operators $\widetilde{S}(z)$, defined by

$$
\begin{aligned}
\widetilde{S}(z) & =S(z)(1-z K S(z))^{-1} \\
& =S(z)\left\{1+\frac{z K S(z)}{1-z \operatorname{tr}(K S(z))}\right\},
\end{aligned}
$$

satisfies the conditions imposed on $\{S(z)\}$ in the preceding paragraph. $\widetilde{U}$ is so defined that $z \in U, \operatorname{Im} z \neq 0$ and $1-z$ trace $(K S(z)) \neq 0$ together imply $z \in \widetilde{U}$.

Suppose further that there exists an increasing sequence $\left\{D_{N}\right\}$ of finite dimensional subspaces of $\mathfrak{H}$, together with a sequence $\left\{T_{N}(z)\right\}$ of operators, defined for $\operatorname{Im} z \neq 0$ and $z \in U$, such that

(a) $P_{N} T_{N}(z) P_{N}=T_{N}(z), P_{N}$ the orthogonal projection onto $D_{N}$

(b) for each set $J$ as in the first paragraph, $T_{N}(z)$ has a representation 


$$
T_{N}(z)=\int_{J} \frac{d \nu_{N}(t)}{z-t}+R_{N}(z)
$$

with $\nu_{N}$ a finite sum of positive masses, each of rank 1 as an operator, and $R_{N}(z)$ holomorphic in $N_{J}$.

(c) $\lim _{N \rightarrow \infty} R_{N}(z)=R(z), z \in N_{J}$ (weak limit)

(d) the total variation of $\nu_{N}$ on $J$ is bounded in $N$, and $\lim _{N \rightarrow \infty}$ $\nu_{N}=\nu$

(e) for almost all $z \in I$ a sesquilinear form $K(z)$ is defined with form domain containing $D, D=\bigcup D_{N}$, and independent of $z$, such that

$$
\left(f, T_{N}(z) K(z) g\right) \longrightarrow(f, g)
$$

as $N \rightarrow \infty$ in measure $(\mu)$ on $I$, for $f \in \mathfrak{F}, g \in$ domain $K(z)$. Then the sequence of operators $\left\{\widetilde{T}_{N}(z)\right\}$ defined by

$$
\widetilde{T}_{N}(z)=T_{N}(z)\left\{1+\frac{z K T_{N}(z)}{1-z \operatorname{tr}\left(K T_{N}(z)\right)}\right\}
$$

stands in a similar relation to the operators $\{\widetilde{S}(z)\}$, and the sesquilinear forms $\widetilde{K}(z)=K(z)-z K$.

Conclusion of proof of Theorem 1 (assuming Theorems 2, 3). To see that Theorems 2 and 3 imply Theorem 1 note first that the conclusions of Theorem 1 are local in $z$, i.e., it suffices to show that each $z_{0} \in I$ is contained in an open interval $I_{0}$ such that the statements of Theorem 1 are valid with $I$ replaced by $I_{0}$. Choose a real number $\sigma$ with $0<\sigma<1$. Then $C(z)=C\left(z_{0}\right)+\left(C(z)-C\left(z_{0}\right)\right)$ with

$$
\left\|C(z)-C\left(z_{0}\right)\right\| \leqslant 1 / 2 \sigma
$$

for $z-z_{0}$ sufficiently small, and, since $C\left(z_{0}\right)$ is compact and symmetric, $C\left(z_{0}\right)=F+\left(C\left(z_{0}\right)-F\right)$ with $\left\|C\left(z_{0}\right)-F\right\| \leqslant 1 / 2 \sigma$, for some symmetric $F$ of finite rank $p$ (say). This gives a splitting of $K(z)$ in a neighborhood $I_{0}$ of $I$

$$
K(z)=K_{1}(z)+z F
$$

With $K_{1}^{\prime}(z)=1+C_{1}(z)$, and $\left\|C_{1}(z)\right\| \leqslant \sigma$ for $\sigma \in I_{0}$. Theorem 2 is applicable to the family $\left\{K_{1}(z)\right\}$. Condition (c) of Theorem 1 implies

$$
1+z S_{1}(z) F=S_{1}(z) K(z)
$$

is invertible for some $z \in N-I$, so $\operatorname{det}\left(1+z S_{1}(z) F\right) \neq 0$ for $z=\omega$ (say).

We claim that we can choose a splitting $F=\sum_{j=1}^{s} F_{j}$ of $F$ as a sum of a finite number of symmetric rank 1 operators $F_{j}$, in such a 
way that

$\operatorname{det}\left(1+\omega S_{1}(\omega) \Sigma_{k}\right) \neq 0$, with $\Sigma_{k}=\sum_{j=1}^{k} F_{j}$, for all $k, 1 \leqslant k \leqslant s$. Theorem 1 is obtained by a finite induction on $k$; at each step one has $\omega \in U$ and $1-\omega$ trace $(K S(\omega)) \neq 0$, so that the first possibility admitted in Theorem 3 does not occur.

To justify the claim made in the preceding paragraph consider the vector space $V$ of symmetric operators $\Sigma$ of finite rank whose range is contained in the range of $F$. The set

$$
D=\left\{\Sigma \mid \operatorname{det}\left(1+\omega S_{1}(\omega) \Sigma\right)=0\right\}
$$

is an algebraic subset of $V$, and $0 \notin D, F \notin D . \quad V$ is a vector space of dimension $(p(p+1)) / 2$ and, since every $\Sigma \in V$ can be written as a sum of $p$ rank 1 operators, it is possible to choose a basis $\left\{E_{i}\right\}$ for $V$ in which each $E_{i}$ has rank 1 . A suitable splitting of $F$ is obtained by choosing a piecewise linear path joining 0 to $F$, each of whose vertices $0=\Sigma_{0}, \Sigma_{1}, \cdots, \Sigma_{s}=F$ does not lie in $D$, and each of whose edges is parallel to one of the basis vectors.

2. Proof of Theorem 2. The proof of Theorems 2 and 3 follows very closely the proof given by Stone [18] of the first part of the spectral theorem for a self-adjoint operator $H$, i.e., the part in which the spectral measure is constructed. The second part of the spectral theorem in which the spectral measure is shown to be projection valued, the projections giving a resolution of the identity, depends upon the Hilbert identity satisfied by the resolvent; here we do not have an analogous identity satisfied by $S(z)$, and therefore we can only assert that the measure constructed is a positive operator valued measure. Stone's method is to approximate $H$ by a sequence $H_{N}$ of symmetric operators of finite rank, and to obtain the matrix elements of the spectral measure of $H$ as limits of the corresponding matrix elements of the spectral measures of the $H_{N}$. That $H_{N}$ has a spectral measure is a reformulation of the spectral theorem for a symmetric operator in a finite dimensional space.

We divide the proofs of Theorems 2 and 3 into a series of lemmas. T2 appearing at the beginning of the statement of a lemma indicates that the notation and hypotheses of Theorem 2 are in force.

Lemma 1, due to Stone, asserts strong convergence of the approximate resolvents constructed from the operators $H_{N}$ to the resolvent of $H$. From it we obtain Lemma 2 which asserts strong convergence of the operators $S_{N}(z)$ to the operator $S(z)$, which plays the role of the resolvent in our Theorem 2. The explicit estimate enables us to verify a condition on the uniformity of the convergence which is essential in the argument which follows. Lemma 3, essen- 
tially due to Rellich, plays the same role in our argument as the spectral theorem for a hermitian operator of finite rank in that of Stone. Lemmas 4, 6, and 8 are elementary lemmas concerning sequences of meromorphic functions $\varphi_{N}(z)$ having simple poles on the real axis; in each case a convergence condition is given for $\operatorname{Im} z \neq 0$ and the conclusion concerns the behavior of the sequence of $z$ real. Lemma 5 gives a basic estimate for the Lebesgue measure of the set on which a meromorphic function of the type described is large. Lemma 7 is an elementary measure theoretic lemma needed in the proof of Theorem 3. Theorem 2 follows from these lemmas and the Stieltjes inversion formula for Hilbert transforms exactly as the first part of the spectral theorem follows from the corresponding assertions in Stone ([18]); this argument is isolated as Lemma 9.

Notice that in Theorem 2 no condition similar to condition (c) in theorem is given or needed. The necessity for this condition appears in Theorem 3, where the possibility arises that $S(z)$ may not exist for any $z$, Theorem 3 asserts that it suffices to demand that $S(z)$ exist for one complex value of $z$. This result follows from the key Lemma 10. The connection between Theorem 1 and the theory of $J$ self-adjoint operators pointed out at the end of $\S 3$ makes it clear that Lemma 10 is best possible. Lemma 11 gives the implication of Lemma 10 for the approximating measures. Theorem 3 then follows from these lemmas and the Stieltjes inversion formula.

Lemma 1. (Stone [18]) Let $H$ be a self-adjoint operator, $z a$ complex number, $\operatorname{Im} z \neq 0$. Let $D=\bigcup_{N=1} D_{N},\left\{D_{N}\right\}$ an increasing sequence of finite dimensional subspaces of $\mathfrak{S}$. Suppose that $D$ is a core for $H$, so that $D$ is contained in the domain of $H$, and $(z-H)$ $D$ is dense in $\mathfrak{S}$. Denote by $T_{N}(z)$ the bounded operator on $\mathfrak{S}$ defined by extending the inverse of $P_{N}(z-H) P_{N}$ on $D_{N}$ to an operator on $\mathfrak{S}$ by setting it equal to zero on the orthogonal complement of $D_{N}$. Then as $N \rightarrow \infty$

$$
T_{N}(z) \longrightarrow(z-H)^{-1} \quad \text { strongly } .
$$

We give the proof because we need not only the result stated but also the estimate given by the proof.

Proof. Given $\varepsilon>0$, and $f \in \mathfrak{S}$, choose $h \in(z-H) D$ so

$$
\|h-f\|<\varepsilon \text {. }
$$

Suppose $h=(z-H) k, k \in D$. Then $k \in D_{N}$ for all $N \geqslant N_{0}$, so

$$
(z-H)^{-1} f=(z-H)^{-1}(f-h)+k
$$




$$
T_{N}(z) f=T_{N}(z)(f-h)+T_{N}(z)(z-H) k
$$

but $k \in D_{N}$ so

$$
T_{N}(z)(z-H) k=T_{N}(z) P_{N}(z-H) P_{N} k=P_{N} k=k .
$$

Also

$$
\left\|(z-H)^{-1}\right\| \leqslant|\operatorname{Im} z|^{-1},\left\|T_{N}(z)\right\| \leqslant|\operatorname{Im} z|^{-1} .
$$

This gives

$$
\left\|(z-H)^{-1} f-T_{N}(z) f\right\| \leqslant 2|\operatorname{Im} z|^{-1}\|f-h\|<2 \varepsilon|\operatorname{Im} z|^{-1} .
$$

REMARK 1. If $L$ is a closed subspace of $\mathfrak{F}$ and $y \in \mathfrak{F}$ we denote by $d(y, L)$ the distance from $y$ to $L$. The above proof gives the estimate

$$
\left\|(z-H)^{-1} f-T_{N}(z) f\right\| \leqslant 2|\operatorname{Im} z|^{-1} d\left(f,(z-H) D_{N}\right) .
$$

Lemma 2. (T2) $K(z)$ has a bounded inverse $S(z)$ in $U-I$. For $z \in U-I$

$$
\lim _{N \rightarrow \infty} T_{N}(z)=S(z) \quad(\text { Strong limit })
$$

Proof. Let $z_{1} \in U$. By supposition $z_{0}=\operatorname{Re} z_{1} \in U$ and the segment $z_{0} z_{1}$ is contained in $U$. Write $H_{0}=H+V\left(z_{0}\right), R\left(z, H_{0}\right)=\left(z-H_{0}\right)^{-1}$. Note that in some neighborhood of $z_{1}$

$$
\sigma\left|z-z_{0}\right|<|\operatorname{Im} z| \text {. }
$$

Then

$$
\begin{aligned}
K(z)^{-1} & =\left(z 1-H_{0}+V(z)-V\left(z_{0}\right)\right)^{-1} \\
& =\left(z 1-H_{0}+\int_{z_{0}}^{z} V^{\prime}(u) d u\right)^{-1} \\
& =\left[1+R\left(z, H_{0}\right) \int_{z_{0}}^{z} V^{\prime}(u) d u\right]^{-1} R\left(z, H_{0}\right) .
\end{aligned}
$$

Since $\left\|R\left(z, H_{0}\right) \int_{z}^{z} V^{\prime}(u) d u\right\| \leqslant \sigma\left|z-z_{0}\right||\operatorname{Im} z|^{-1}<1$ in a neighborhood of $z_{1}$, the inverse of the first factor in (2.2) exists and may be computed as a geometric series. Since $\left(V(z)-V\left(z_{0}\right)\right)\left(z-z_{0}\right)^{-1}$ is holomorphic in $z$, the resulting representation of $S(z)=K(z)^{-1}$ as a norm convergent series whose terms are holomorphic establishes that $S(z)$ exists and is holomorphic in a neighborhood of $z-z_{1}$.

Finally the approximation theorem (2.1) follows from Lemma 1 and the following observations: 
1. If $A_{N} \rightarrow A, B_{N} \rightarrow B$ strongly as $N \rightarrow \infty$, and $\left\{A_{N}\right\}$ is norm bounded, then $A_{N} B_{N} \rightarrow A B$ strongly.

2. If $A_{N, k} \rightarrow A_{k}$ strongly as $N \rightarrow \infty$ (for all $k$ ), and $\left\|A_{N, k}\right\| \leqslant c_{k}$ with $\Sigma c_{k}<\infty$ then $S_{N}=\sum_{k} A_{N, k} \rightarrow S=\sum_{k} A_{k}$ strongly.

3. Since $D$ is a core for $H$ and $V(\operatorname{Re} z)$ is bounded hermitian, $D$ is a core for $K(\operatorname{Re} z)=-H-V(\operatorname{Re} z)+\operatorname{Re} z 1$.

REMARK 2. From the explicit estimate given in Remark 1, we obtain

$$
\left\|S(z) f-T_{N}(z) f\right\| \leqslant \frac{2|\operatorname{Im} z|^{-1}}{(1-\sigma)^{2}} d\left(f,[K(\operatorname{Re} z)+i \operatorname{Im} z] D_{N}\right) .
$$

LEMma 3. (T2) $T_{N}(z)$ is meromorphic in U. Its poles are on the real axis; they are simple and the residues are positive (operators).

Proof. For $z$ real, write $H(z)=H+V(z)$. $T_{N}(z)$ may be considered as the extension to $\mathfrak{S}$ of the resolvent $R\left(\omega, P_{N} H(z) P_{N}\right)$ of $P_{N} H(z) P_{N}$ (considered as a hermitian operator on $D_{N}$ ), evaluated at $\omega=z$. By the spectral theorem

$$
R\left(\omega, P_{N} H(z) P_{N}\right)=\sum_{i} \frac{P_{N}^{i}(z)}{\omega-\lambda_{N}^{i}(z)} .
$$

By the argument used in the proof of Lemma 2, $R\left(\omega, P_{N} H(z) P_{N}\right)$ may be shown to be holomorphic as a function of two variables for $z$ and $\omega$ in $U$ with $|\operatorname{Im} \omega|>\sigma|\operatorname{Im} z|$; in particular, $T_{N}(z)$ is holomorphic for $z \in U,|\operatorname{Im} z| \neq 0$. By a theorem of Rellich ([9], p. 120) the eigenvalues $\lambda_{N}^{i}(z)$ are holomorphic in a neighborhood of $I$, as also are the projectors $P_{N}^{i}(z)$. It follows then, from (2.3) with $\omega=z$, that $T_{N}(z)$ is meromorphic in a neighborhood of $I$ (and so in all of $U$ ) with its only poles at points of $I$ such that $z-\lambda_{N}^{i}(z)=0$ for some $i . \lambda_{N}^{i}(z)$ has derivative trace $\left(V(z) P_{N}^{i}(z)\right)$, which is bounded in absolute value by $\sigma$. Thus $d / d z\left(z-\lambda_{N}^{i}(z)\right) \geqslant 1-\sigma>0$ for $z$ real, showing that $z-\lambda_{N}^{i}(z)=0$ for at most one value of $z \in I$, say for $z=\nu_{N}^{i}$. The corresponding pole is simple with a positive residue (a positive multiple of $\left.P_{N}^{i}\left(\nu_{N}^{i}\right)\right)$.

The following lemmas will allow us to pass from the approximation theorem off the real axis given by Lemma 2 to the type of approximation on the real axis which appears in Theorems 2 and 3.

LEMMA 4. Let $I$ be a finite open interval of the real axis, $U$ a complex neighborhood of $I$. Suppose that $\left\{\varphi_{N}(z)\right\}$ is a sequence of functions meromorphic in $U$. For each $N$ suppose that $\varphi_{N}(z)$ has 
only simple poles and that these lie on the real axis. For $J$ any open interval with closure contained in $I$ denote by $C_{N}(J)$ the sum of the absolute values of the residues of the poles of $\varphi_{N}(z)$ in $J$.

Suppose that $(\operatorname{Im} z)^{k} \varphi_{N}(z) \rightarrow 0$ as $N \rightarrow \infty$ uniformly on compacts contained in $U$, for some positive integer $k$; and that the residues of $\varphi_{N}(z)$ are all positive. Then for any $J$ as above, $\lim _{N} C_{N}(J)=0$.

Similarly, if $\left|(\operatorname{Im} z)^{k} \varphi_{N}(z)\right|$ is bounded, uniformly on compacts in $U$, and the residues of $\varphi_{N}(z)$ are all positive then, for any $J, C_{N}(J)$ is bounded uniformly in $N$.

Proof. We omit the simple proof.

The next lemma is the starting point for the classical theory of the Hilbert transform.

Lemma 5. Suppose $c_{i}>0$, and $z_{i}$, are real numbers defined for $1 \leqslant i \leqslant m$. Let $\delta>0$. Set $f(\boldsymbol{z})=\sum_{i=1}^{m} c_{i} /\left(z-z_{i}\right)$. Then

$$
\mu\{z|| f(z) \mid \geqslant \delta\}=\frac{2}{\delta}\left(\sum_{i=1}^{m} c_{i}\right) .
$$

COROLlary. Let the $c_{i}$ be complex, and $f(z)$ defined (for z real) as before. The $\mu\{z|| f(z) \mid \geqslant \delta\} \leqslant 16 / \delta\left(\sum_{i=1}^{m} c_{i}\right)$.

Proof. For the proof of Lemma 5 and its corollary see [20].

(Lemma 5 can be stated in terms of $g(z)=[f(z)]^{-1}: g(z)$ regarded as a transformation of the line onto itself is (Lebesgue) measure preserving if $\Sigma c_{i}=1$. But the point of view suggested by this formulation does not seem to have been developed.)

LEMma 6. We retain the notation and hypotheses of the first paragraph of Lemma 4. Suppose

(a) $(\operatorname{Im} z)^{k} \varphi_{N}(z) \rightarrow 0$ as $N \rightarrow \infty$ uniformly on compacts contained in $U$, for some positive integer $k$

(b) for any $J, \lim _{N \rightarrow \infty} C_{N}(J)=0$.

Then as $N \rightarrow \infty, \varphi_{N}(z) \rightarrow 0$ in measure $(\mu)$ on $I$.

Proof. It suffices to show that for each open interval $V$ whose closure is contained in $I$, as $N \rightarrow \infty \varphi_{N}(z) \rightarrow 0$ in measure on $V$.

Suppose $V=(c, d)$. (a) implies $[(z-c)(d-z)]^{k} \varphi_{N}(z) \rightarrow 0$ as $N \rightarrow \infty$ uniformly on the boundary of a rectangle $R$ with vertices $c \pm i \varepsilon$, $d \pm i \varepsilon, \varepsilon>0$ sufficiently small that it is contained in $U$. Let

$$
\varphi_{N}(z)=\psi_{N}(z)+\tau_{N}(z)
$$


be the splitting of $\varphi_{N}(z)$ into the sum $\psi_{N}(z)$ of the principal parts at its poles in $V$, and $\tau_{N}(z)$ holomorphic in $V$. From (b) it follows that $[(z-c)(d-z)]^{k} \psi_{N}(z) \rightarrow 0$ as $N \rightarrow \infty$ uniformly on the boundary of $R$. Hence also $[(z-c)(d-z)]^{k} \tau_{N}(z) \rightarrow 0$ as $N \rightarrow \infty$ uniformly on the boundary of $R$. By the maximum modulus principle

$$
[(z-c)(d-z)]^{k} \tau_{N}(z) \longrightarrow 0
$$

as $N \rightarrow \infty$ uniformly in the interior of $R$ and in particular on $V$. Thus $\tau_{N}(z) \rightarrow 0$ in measure $(\mu)$ on $V$. But (b) and the corollary to Lemma 5 imply $\psi_{N}(z) \rightarrow 0$ in measure $(\mu)$ on $V$. Hence $\varphi_{N}(z) \rightarrow 0$ in measure $(\mu)$ on $V$.

Definition. A sequence $\left\{\varphi_{N}\right\}$ of measurable functions on a measure space $M=(X, \boldsymbol{R}, m)$ is bounded in measure if

$$
\forall \varepsilon>0 \quad \exists K=K(\varepsilon)
$$

and

$$
N_{0}=N_{0}(\varepsilon) \ni m\left\{x|| \varphi_{N}(x) \mid>K\right\} \leqslant \varepsilon \quad \forall N \geqslant N_{0} \text {. }
$$

The definition is motivated by

LEMma 7. A sequence $\left\{\varphi_{N}\right\}$ of measurable functions on $M$ is bounded in measure if and only if, for every sequence $\left\{f_{N}\right\}$ of measurable functions converging to zero in measure on $M$, the sequence $\left\{f_{N} \varphi_{N}\right\}$ also converges to zero in measure on $M$.

Proof. We omit the proof.

Note that if, for some subsequence $\left\{N_{j}\right\}$, the functions $\left\{\left(\varphi_{N_{j}}(x)\right)^{-1}\right\}$ converge to zero in measure on a set of strictly positive measure, then the sequence $\left\{\phi_{N}\right\}$ is not bounded in measure. However, the converse is false. Counterexample: Take $M=[0,1]$, with Lebesgue measure $\mu$. Consider the sets

$$
E_{N}=\{x \mid \text { the Nth binary digit of } x=1\} \text {. }
$$

Set $\varphi_{N}(x)=N$ for $x \in E_{N}, \varphi_{N}(x)=1$ for $x \notin E_{N}$.

LEMMA 8. We retain the notation and hypotheses of the first paragraph of Lemma 4. Suppose

(a) $\left|(\operatorname{Im} z)^{k} \varphi_{N}(z)\right|$ is bounded as $N \rightarrow \infty$ uniformly on compacts contained in $U$, for some positive integer $k$

(b) for any $J, C_{N}(J)$ is bounded as $N \rightarrow \infty$. Then as $N \rightarrow \infty, \varphi_{N}(z)$ is bounded in measure $(\mu)$ on $I$. 
Proof. The proof is essentially the same as that of Lemma 6 .

Note that, by Lemma 4, condition (b) can be replaced in Lemma 6 and in Lemma 8 by the condition that the residues of $\varphi_{x}(z)$ in $J$ be positive.

Proof of the second part of Theorem 2. We can now prove the on-axis approximation (1.3) in Theorem 2. Write

$$
\varphi_{N}(z)=\left(f, T_{N}(z) K(z) g\right)-(f, g)
$$

for $f \in \mathfrak{F}, g \in$ domain of $H$. Lemma 3 shows that $\varphi_{N}(z)$ is meromorphic in $U$ with simple poles on the real axis and positive residues. Lemma 2 shows that condition (a) of Lemma 6 is satisfied. In fact the remark following Lemma 2 gives

$$
\left|\varphi_{N}(z)\right|(\operatorname{Im} z)^{2} \leqslant \frac{2|\operatorname{Im} z|}{(1-\sigma)^{2}}\|f\| d\left(K(z) g,[K(\operatorname{Re} z)+i \operatorname{Im} z] D_{N}\right) .
$$

The right side of this inequality is continuous in $z$ for $z \in U$ and decreases monotonically to zero as $N \rightarrow \infty$ for each fixed $z$, so by Dini's theorem the convergence is uniform on compacts contained in $U$. $(K(\operatorname{Re} z) D$ may not be dense, so we do not know whether for $z$ real $d\left(K(z) g, K(z) D_{N}\right) \rightarrow 0$ as $N \rightarrow \infty$; for this reason an additional factor $|\operatorname{Im} z|$ has been multiplied into the inequality.) It remains to verify condition (b) of Lemma 6.

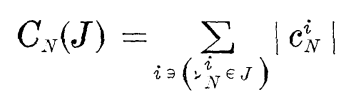

where $c_{N}^{i}$ is the residue of $\varphi_{N}(z)$ at the pole $\nu_{N}^{i}$. We saw in the proof of Lemma 3 that $c_{N}^{i}$ has the form

$$
c_{N}^{i}=\tau_{N}^{i}\left(f, P_{N}^{i}\left(\nu_{N}^{i}\right)\left[K\left(\nu_{N}^{i}\right)-P_{N} K\left(\nu_{N}^{i}\right) P_{N}\right] g\right)
$$

where $\tau_{N}^{i}$ is positive and $\leqslant(1-\sigma)^{-1}$. Schwarz's inequality gives

$$
C_{N}(J) \leqslant\left[A_{N}(J) B_{N}(J)\right]^{1 / 2}
$$

with

$$
\begin{aligned}
& A_{Y}(J)=\sum_{i \in\left(\dot{\nu}_{N}^{i} \in J\right)}\left\|P_{N}^{i}\left(\nu_{N}^{i}\right) f\right\|^{2} \tau_{. V}^{i} \\
& B_{V}(J)=\sum_{i \in\left(\nu_{N}^{i} \in J\right)}\left\|P_{N}^{i}\left(\nu_{N}^{i}\right)\left(K\left(\nu_{N}^{i}\right)-P_{N} K\left(\nu_{N}^{i}\right) P_{N}\right) g\right\|^{2} \tau_{. V}^{i} \cdot
\end{aligned}
$$

We claim $A_{x}(J)$ is bounded, and $B_{N}(J) \rightarrow 0$ as $N \rightarrow \infty$. Consider the functions $\left(f, T_{1}(z) f\right)$. These functions are meromorphic in $U$ with 
simple poles at the points $\nu_{N}^{i}$. The residues at these poles are positive, and $A_{N}(J)$ is the sum of the residues at poles in $J .(\operatorname{Im} z)\left(f, T_{N}(z) f\right)$ is bounded on compacts in $U$, uniformly in $N$, so by Lemma $4, A_{N}(J)$ is bounded in $N$.

Next consider the functions

$$
\lambda_{N}(z)=\left(g,\left(K(z)-P_{N} K(z) P_{N}\right) T_{N}(z)\left(K(z)-P_{N} K(z) P_{N}\right) g\right) .
$$

These are meromorphic in $U$ with simple poles at the points $\nu_{N}^{i}$. The residues at these poles are positive, and $B_{N}(J)$ is the sum of the residues at poles in $J$. Since $\left(g,\left(K(z)-P_{N} K(z) P_{N}\right) g\right)$ is holomorphic in $U, B_{N}(J)$ is also the sum of the residues at poles in $J$ of

$$
\begin{aligned}
\bar{\lambda}_{N}(z) & =\lambda_{N}(z)+\left(g,\left(K(z)-P_{N} K(z) P_{N}\right) g\right) \\
& =\left(g, K(z) T_{N}(z)\left(K(z)-P_{N} K(z) P_{N}\right) g\right) .
\end{aligned}
$$

Now

$$
\left|\bar{\lambda}_{N}(z)\right| \leqslant\|K(z) g\|\left\|T_{N}(z)\left(K(z)-P_{N} K(z) P_{N}\right) g\right\| \bullet
$$

The first factor is bounded on compacts in $U$, and the second factor is (by Lemma 2$) \leqslant 2 /\left((1-\sigma)^{2}\right)|\operatorname{Im} z|^{-1} d\left(K(z) g,[K(\operatorname{Re} z)+i \operatorname{Im} z] D_{N}\right)$, and so, like $\left|\varphi_{N}(z)\right|$, satisfies condition (a) of Lemma 6. Lemma 4 is thus applicable to the functions $\bar{\lambda}_{N}(z)$, and implies $\lim B_{N}(J)=0$, $N \rightarrow \infty$.

We have now shown $C_{N}(J) \rightarrow 0$ as $N \rightarrow \infty$. The convergence to zero in measure $(\mu)$ on $I$ of the functions $\varphi_{N}(z)$ now follows from Lemma 6.

Proof of the first part of Theorem 2. To complete the proof of Theorem 2, it remains to obtain the representation (1.2) for $S(z)$. The argument used by Stone [18] (Chapter 5, §2) carries over without essential change.

The argument rests on the weak* compactness of a bounded set of Borel measures, and on the Stieltjes inversion formula. We recall this formula for the reader's convenience: Let $\nu$ be a Borel measure on $R$,

$$
\widetilde{\nu}(z)=\int \frac{d \nu(t)}{z-t}
$$

$(\operatorname{Im} z \neq 0)$ its Hilbert transform, $F(t)=\nu((-\infty, t])$ the distribution function of $\nu$. Then $F$ (and hence $\nu$ ) may be recovered from $\tilde{\nu}$ by means of the formula

$$
F(b)-F(a)=\lim _{\varepsilon \rightarrow 0+} \frac{1}{2 \pi i} \int_{C_{s}} \tilde{\nu}(z) d z .
$$


Here $a$ and $b$ are points of continuity of $F$, and $C_{\varepsilon}$ is a contour obtained from a positively oriented rectangle with vertices $a-i, b-i$, $b+i$, and $a+i$ by deleting the segments of its boundary in the strip $|\operatorname{Im} z| \leqslant \varepsilon$.

It suffices for all $x, y \in \mathfrak{S}$ and every interval $J$ whose closure is contained in $I$, to obtain a decomposition

$$
(x, S(z) y)=\int_{J} \frac{d \nu_{x, y}(t)}{z-t}+R_{x, y}^{J}(z)
$$

of $(x, S(z) y)$ as the sum of the Hilbert transform of a Borel measure $\nu_{x, y}$ on $J$, positive for $x=y$, and a function $R_{x, y}^{J}(z)$ holomorphic in $U-(I-J)$. For the total variation of $\nu_{x, y}$ on $J$ we must have a bound

$$
\left\|\nu_{x, y}\right\|_{J} \leqslant M(J)\|x\|\|y\|
$$

and $\nu_{x, y}$ must be shown to be sesquilinear in $x, y$. We do not put a subscript $J$ on $\nu_{x, y}$ because the Stieltjes inversion formula shows that if $J_{1} \subset J_{2}$, and we have such a representation of $(x, S(z) y)$ for both $J_{1}$ and $J_{2}$ then $\boldsymbol{\nu}_{x, y}^{J_{1}}$ is the restriction to $J_{1}$ of $\boldsymbol{\nu}_{x, y}^{T_{2}}$.

The decomposition for $(x, S(z) y)$ is obtained from the corresponding decompositions for the approximations $\left(x, T_{N}(z) y\right)$ by an application of Lemma 9 (below). We have seen (Lemma 3) that $T_{N}(z)$ is meromorphic in $U$ with simple poles on the real axis having positive residues. By splitting $\left(x, T_{N}(z) y\right)$ into the sum of the principal parts at the poles in $J$ and a function $R_{x, y}^{N, J}(z)$ holomorphic in $U-(I-J)$, we obtain the desired decomposition of $\left(x, T_{N}(z) y\right)$. The measure $\nu_{x, y}^{N}$ is just the discrete measure supported by the poles of $\left(x, T_{N}(z) y\right)$, which assigns to each pole its residue. $\left\|\nu_{x, y}^{N}\right\|_{J}$ is the sum of the absolute values of the residues at poles in $J$, and for this we obtain a uniform bound of the form $M(J)\|x\|\|y\|$ as in the proof of (1.3). We note that for $x=y$ the residues are positive. The conditions of Lemma 9 are thus satisfied by the functions $\varphi_{N}(z)=\left(x, T_{N}(z) y\right)$ for any $x, y \in \mathfrak{F}$ and the resulting measures $\nu_{x, y}$ depend on $x, y$ in the way described in the preceding paragraph.

This completes the proof of Theorem 2 .

Lemma 9. Let $I$ be a finite open interval of the real axis, $V$ a complex neighborhood of $I$. Let $\varphi_{N}(z)$ be a sequence of functions defined and holomorphic in $V-I$. Suppose that

(a) $\left|\varphi_{N}(z)\right| \leqslant K|\operatorname{Im} z|^{-k}$ for all $N$, some integer $k$ and some $K>0$, uniformly in $z$ on compacts contained in $V$

(b) $\varphi_{N}(z) \rightarrow \varphi(z)$ as $N \rightarrow \infty$, pointwise in $V-I$

(c) for every interval $J$, whose closure is contained in $I$, there 
are positive measures $\nu_{N, J}$ on $J$, and functions $\psi_{N, J}(z)$, holomorphic in $V-(I-J)$, such that $\varphi_{N}(z)=\widetilde{\nu}_{N, J}(z)+\psi_{N, J}(z)$.

Then, for each such $J$, the measures $\nu_{N, J}$ converge as $N \rightarrow \infty$ to a measure $\nu_{J}$, and $\psi(z)=\varphi(z)-\widetilde{\nu}_{J}(z)$ admits a holomorphic extension to $V-(I-J)$.

Proof. From (a) we obtain a bound $\left\|\nu_{N, J}\right\| \leqslant B$ for all $N$. By weak* compactness of bounded sets of measures, we obtain a subsequence of $\nu_{N, J}$ converging as $N \rightarrow \infty$ to $\nu_{J}$ (say). Define

$$
\psi(z)=\varphi(z)-\widetilde{\nu}_{J}(z) \text {. }
$$

Then $\widetilde{\nu}_{N_{j}}(z) \rightarrow \widetilde{\nu}_{J}(z)$ for $z \in V-I$, and so $\psi_{N_{j}}(z) \rightarrow \psi(z)$ as $j \rightarrow \infty$. If $J=(a, b)$, we can choose a rectangle $R$ with vertices $a \pm i \varepsilon, b \pm i \varepsilon$ contained in $V$, and show that the functions $(b-z)^{k}(z-a)^{k} \psi_{N_{j}}(z)$ converge uniformly on the boundary of $R$ and hence also in its interior. Thus $\psi(z)$ admits a holomorphic extension $\lim _{j \rightarrow \infty} \psi_{N_{j}}(z)$ to $V-(I-J)$. Finally, we note that, since $\nu_{J}$ can be recovered uniquely from $\varphi(z)$ by the Stieltjes inversion formula, the passage to a subsequence of $\nu_{N, J}$ was not necessary, and, in fact, $\nu_{N, J} \rightarrow \nu_{J}$ as $N \rightarrow \infty$.

3. Proof of Theorem 3. If $\nu$ is a Borel measure on the real line with compact support, we denote by

$$
\widetilde{\nu}(z)=P \cdot v \cdot \int \frac{d \nu(t)}{z-t}
$$

its Hilbert transform. $\tilde{\nu}(z)$ is holomorphic in $z$ for $z$ not in the support of $\nu$, and (see [20]) exists almost everywhere ( $\mu$ ) (as a principal value integral) for $z$ in the support of $\nu$. We wite $\|\boldsymbol{\nu}\|$ for the total mass of $\nu$.

LEMmA 10. Let $\nu$ be a positive Borel measure on the real line with compact support, and $g(z)$ a function holomorphic in the connected open set $U$ of the complex plane. Suppose $U$ is symmetric with respect to the real axis, and $g(\bar{z})=\overline{g(z)}, z \in U$. Exclude the trivial case: ע a measure whose support does not intersect $U$,

$$
\widetilde{\nu}(z)+g(z)=0
$$

identically. Define

$$
\begin{aligned}
E=E(\nu, g)= & \{z \in U \mid \text { either } \operatorname{Im} z \neq 0 \text { and } \widetilde{\nu}(z)+g(z)=0 \text { or } \\
& \left.\operatorname{Im} z=0, g^{\prime}(z) \geqslant \int \frac{d \nu(t)}{(z-t)^{2}}, \text { and } \widetilde{\nu}(z)+g(z)=0\right\} .
\end{aligned}
$$


Then $E$ is discrete. If $g$ is a polynomial of degree $\leqslant 2 m, E$ contains at most $m$ points in $\operatorname{Im} z \geqslant 0$.

Proof. Let $z_{1}, \cdots, z_{m}$ be $m$ (distinct) points of $E \cap\{z \mid \operatorname{Im} z \geqq 0\}$, $C$ a simple closed contour in $U$ containing these points and their complex conjugates in its interior. Write $D(t)=\prod_{i=1}^{m}\left|z_{i}-t\right|^{2}$. Cauchy's residue theorem and the definition of $E$ give the inequality

$$
\int_{R} \frac{d \nu(t)}{D(t)} \leqslant \frac{1}{2 \pi i} \int_{C} \frac{g(z)}{D(z)} d z
$$

(for $z$ complex $D(z)$ is defined as $\prod_{i=1}^{m}\left(z_{i}-z\right)\left(\bar{z}_{i}-z\right)$ ).

If $g(z)$ is a polynomial of degree $\leqq 2 m-2$, the right side of (3.1) is zero (since $D(z)$ has degree $2 m$ ). (3.1) then forces $\nu$ to be the zero measure. $z_{1}, \cdots, z_{m}$ are then zeros of $g$, which implies $g$ is identically zero, and we have the trivial case. This proves the final assertion of Lemma 10.

Now return to the general case: $g(z)$ holomorphic. Clearly any limit point $z_{0}$ of $E$ must lie on the real axis in the support of $\nu$. For otherwise, $\tilde{\nu}(z)$ would be holomorphic in a neighborhood of $z_{0}$ and so $\tilde{\nu}(z)+g(z)$ would vanish identically, and we would have the trivial case. In (3.1) split $g$ into the sum of the first $2 m$ terms of its Taylor series about $z_{0}$, and a remainder $R_{m}(z)$. This gives

$$
\int_{R} \frac{d \nu(t)}{D(t)} \leqslant \frac{g^{(2 m-1)}\left(z_{0}\right)}{(2 m-1) !}+\frac{1}{2 \pi i} \int_{C} \frac{R_{m}(z)}{D(z)} d z
$$

Since $z_{0}$ is a limit point of $E$, we may for any $\varepsilon>0$ choose the $m$ points to lie within the circle $\left|z_{0}-z\right|=\varepsilon$. We then take for $C$ the circle $\left|z-z_{0}\right|=2 \varepsilon$. For $R_{m}(z)$ we have an estimate

$$
\left|R_{m}(z)\right| \leqslant K\left|z-z_{0}\right|^{2 m}
$$

in some fixed circle with center $z_{0}$. The second term on the right of (3.2) is thus $0(\varepsilon)$. Taking the limit $\varepsilon \rightarrow 0$ we obtain by Fatou's lemma

$$
\int \frac{d \nu(t)}{\left(z_{0}-t\right)^{2 m}} \leqslant \frac{g^{(2 m-1)}\left(z_{0}\right)}{(2 m-1) !}
$$

We claim (3.3) implies that $z_{0}$ is not in the support of $\nu$. This contradiction will complete the proof of Lemma 10 .

Consider for $x$ real the series $\sum_{m=0}^{\infty} x^{2 m} /\left(\left(z_{0}-t\right)^{2 m}\right)$. For $|x|$ sufficiently small, (3.3) implies that its partial sums are $\nu$ integrable as functions of $t$, and gives a uniform bound for the $\nu$-integral of the $m$ th partial sum. By Fatou's lemma the sum of the series is $\nu$ integrable. But for $|x|>\left|z_{0}-t\right|$ the series diverges. The interval 
$\left|z_{0}-t\right| \leqslant|x| / 2$ therefore does not intersect the support of $\nu$.

Note that Lemma 5 implies that if $\left\{\nu_{N}\right\}$ is a bounded sequence of positive measures on the real line, each a finite sum of point masses, the sequence $\left\{\tilde{\nu}_{N}(z)\right\}$ of their Hilbert transforms is bounded in measure. The following Lemma 11 may therefore be considered as a generalization of Lemma 5 .

Lemma 11. Let $\left\{\boldsymbol{\nu}_{N}\right\}$ be a bounded sequence of positive measures on the real line, each a finite sum of point masses. Suppose that for each $N$ the support of $\nu_{N}$ is contained in some finite interval $I$ (independent of $N$ ). Let $g(z)$ be a function holomorphic in the open set $U$ of the complex plane. Suppose $U$ symmetric with respect to the real axis, and $g(\bar{z})=\overline{g(z)}, z \in U$. Then either some subsequence of $\left\{\nu_{N}\right\}$ converges to a positive measure $\nu$ such that $\widetilde{\nu}(z)+g(z)=0$ identically, or the sequence $\varphi_{N}(z)=\left(\widetilde{\mathcal{V}}_{N}(z)+g(z)\right)^{-1}$ is bounded in measure $(\mu)$ on $U \cap \boldsymbol{R}$.

Proof. A sequence of functions is bounded in measure $(\mu)$ if and only if every subsequence contains a subsequence with this property. By compactness of a bounded set of measures supported by $I$, every subsequence of $\left\{\boldsymbol{\nu}_{N}\right\}$ contains a subsequence which converges weakly. To prove that $\left\{\varphi_{N}\right\}$ is bounded in measure $(\mu)$ on $U$, it therefore suffices to prove that for every subsequence of $\left\{\boldsymbol{\nu}_{N}\right\}$ which converges, the corresponding subsequence of $\left\{\varphi_{N}\right\}$ is bounded in measure on $U \cap \boldsymbol{R}$. So we may as well assume at the outset that, as $N \rightarrow \infty$, $\nu_{N}$ converges to some positive measure $\nu$.

For $\varepsilon>0$, and $J$ a finite closed interval contained in $U$, set

$$
G_{\varepsilon}^{N}=\left\{x \in J|| \varphi_{N}(x) \mid \geqslant \varepsilon^{-1}\right\} .
$$

We have to show that for every $J$

$$
\lim _{\varepsilon \rightarrow 0}\left(\varlimsup_{N \rightarrow \infty} \mu\left(G_{\varepsilon}^{N}\right)\right)=0 .
$$

Denote by $E$ the set defined in Lemma 10 . Since $E$ is discrete it suffices to prove (3.4) for $J$ a closed interval not intersecting $E$. With this condition on $J$, we claim that, for some $\varepsilon_{0}>0$ and $B>0$, the functions

$$
\varphi_{N, \alpha}(z)=\left(\widetilde{\nu}_{N}(z)+g(z)-\alpha\right)^{-1},
$$

for sufficiently large $N$, satisfy

$$
\left|\varphi_{N, \alpha}(z)\right| \leqslant B|\operatorname{Im} z|^{-1}
$$

for all real $\alpha$ such that $|\alpha| \leqslant \varepsilon_{0}$ and $z$ in some neighborhood $V$ of $J$. 
If not, for some $x \in J$ we will have sequences $z_{n}$ of nonreal numbers converging to zero, $\alpha_{n}, \beta_{n}$ of real numbers converging to zero, and $N_{n}$ of positive integers $\rightarrow \infty$ such that

$$
\begin{gathered}
\int \frac{d \nu_{N_{n}}(t)}{\left|z_{n}-t\right|^{2}}=\frac{\operatorname{Im} g\left(z_{n}\right)}{\operatorname{Im} z_{n}}+\beta_{n} \\
\int \frac{\left(\operatorname{Re} z_{n}-t\right)}{\left|z_{n}-t\right|^{2}} d \nu_{N_{n}}(t)+\operatorname{Re} g\left(z_{n}\right)-\alpha_{n}=0 .
\end{gathered}
$$

From (3.5) we have, for any $\delta>0$, and all $n$

$$
\int_{|x-t\rangle \geqslant 0} \frac{d \nu_{N_{n}}(t)}{\left|z_{n}-t\right|^{2}} \leqslant \frac{\operatorname{Im} g\left(z_{n}\right)}{\operatorname{Im} z_{n}}+\beta_{n} .
$$

As $n \rightarrow \infty$ the left side of (3.7) converges to

$$
\int_{|x-t| \geqslant o} \frac{d \nu(t)}{(x-t)^{2}}
$$

For, if $n$ is sufficiently large, $\left|x-\operatorname{Re} z_{n}\right| \leqslant \delta / 2$, and then

$$
\begin{aligned}
& \int_{|x-t| \geqslant 0}\left\{\frac{1}{\left|z_{n}-t\right|^{2}}-\frac{1}{|x-t|^{2}}\right\} d \nu_{N_{n}}(t) \\
\leqslant & \left\|\nu_{N_{n}}\right\| \sup _{t \ni|x-t| \geqslant 0}\left\{\frac{1}{\left|z_{n}-t\right|^{2}}-\frac{1}{|x-t|^{2}}\right\}
\end{aligned}
$$

$\rightarrow 0$ as $n \rightarrow \infty$, since the measures $\nu_{N}$ are uniformly bounded, while

$$
\int_{|x-t| \geqslant 0} \frac{d \nu_{N_{n}}(t)}{|x-t|^{2}} \longrightarrow \int_{|x-t| \geqslant 0} \frac{d \nu(t)}{|x-t|^{2}}
$$

as $n \rightarrow \infty$, since $\nu_{N} \rightarrow \nu$ as $N \rightarrow \infty$.

The right side of (3.7) converges to $g^{\prime}(x)$ as $n \rightarrow \infty$, so taking first the limit $n \rightarrow \infty$, and then the limit $\delta \rightarrow 0$ we obtain

$$
\int \frac{d \nu(t)}{|x-t|^{2}} \leqslant g^{\prime}(x)
$$

From (3.8) it follows that $\widetilde{\nu}(x)$ exists. For $\delta>0$ denote by $\nu_{\hat{o}}\left(\nu_{N, \hat{o}}\right)$ the restriction of $\nu\left(\nu_{N}\right)$ to $|x-t| \geqslant \delta$. Then

$$
\begin{aligned}
\left|\widetilde{\mathcal{\nu}}(x)-\operatorname{Re} \widetilde{\nu}_{N_{n}}\left(z_{n}\right)\right| \leqslant\left|\widetilde{\nu}(x)-\widetilde{\nu}_{\hat{\delta}}(x)\right| & +\left|\operatorname{Re} \widetilde{\nu}_{N_{n}}\left(z_{n}\right)-\operatorname{Re} \widetilde{\nu}_{N_{n}, \hat{o}}\left(z_{n}\right)\right| \\
& +\left|\widetilde{\nu}_{\hat{o}}(x)-\operatorname{Re} \widetilde{\nu}_{N_{n}, \hat{o}}\left(z_{n}\right)\right| \\
& \leqslant \delta \int \frac{d \nu(t)}{(x-t)^{2}}+\delta \int \frac{d \nu_{N_{n}}(t)}{\left|z_{n}-t\right|^{2}}+\left|\widetilde{\nu}_{\hat{o}}(x)-\operatorname{Re} \widetilde{\nu}_{N_{n}, \hat{o}}\left(z_{n}\right)\right| \\
\leqslant & \leqslant \delta \int \frac{d \nu(t)}{(x-t)^{2}}+\left|\widetilde{\nu}_{\hat{o}}(x)-\operatorname{Re} \widetilde{\nu}_{N_{n}, \hat{o}}\left(z_{n}\right)\right|
\end{aligned}
$$


for sufficiently large $n$. As $n \rightarrow \infty$ the second term in (3.9) $\rightarrow 0$ by the convergence of $\nu_{N}$ to $\nu$. Since $\delta>0$ was arbitrary we obtain

$$
\begin{aligned}
\widetilde{\nu}(x) & =\lim \operatorname{Re} \widetilde{\nu}_{N_{n}}\left(z_{n}\right) & & \text { as } n \rightarrow \infty \\
& =-g(x) & & (\text { from }(3.6))
\end{aligned}
$$

(3.8) and (3.10) show $x \in E$; but this contradicts the choice of $J$. Next we assert that for sufficiently small $\varepsilon$, and large $N$,

$$
\widetilde{\mathcal{\nu}}_{N}^{\prime}(x)+g^{\prime}(x)<0 \quad \text { for all } x \in G_{\varepsilon}^{N} \text {. }
$$

For, if not, we have, for some sequences $N_{n}$ of positive integers $\rightarrow \infty$, and $x_{n}$ of points of $J$ converging to $x \in J$

$$
\begin{aligned}
\lim _{n \rightarrow \infty} \mid & \widetilde{\nu}_{N_{n}}\left(x_{n}\right)+g\left(x_{n}\right) \mid=0 \\
& \widetilde{\nu}_{N_{n}}^{\prime}\left(x_{n}\right)+g^{\prime}\left(x_{n}\right) \geqslant 0 .
\end{aligned}
$$

But then, by essentially the same argument as in the preceding paragraph, we can show $x \in E$, and this contradicts the choice of $J$.

We have established that, for some neighborhood $V$ of $J$, the functions $\varphi_{N}(z)$ are meromorphic in $V$, having simple poles on the real axis with negative residues (the residue of $\varphi_{N}(z)$ at $z=x$ is $\left.\left(\widetilde{\mathcal{V}}_{N}^{\prime}(x)+g^{\prime}(x)\right)^{-1}\right)$, and satisfy $\left|\varphi_{N}(z)\right| \leqslant B|\operatorname{Im} z|^{-1}$ in $V$, i.e., that in $V$ they satisfy the conditions of the first and final paragraphs of Lemma 4. By Lemma 8 and the remark which immediately follows Lemma 8 , the sequence $\varphi_{N}(z)$ is bounded in measure $(\mu)$ on $J$.

Lemma 9 gives the following corollary to Lemma 11.

Lemma 12. Let $g(z), U, \nu$ be as in Lemma 10, and $E$ the discrete subset of $U$ defined in Lemma 10. Then on each interval $J$ contained in $U \cap \boldsymbol{R}$ whose closure does not intersect $E$, there exists a positive measure $\tau_{J}$ such that in some complex neighborhood $V_{J}$ of $J$

$$
\tau_{J}(z)+(\tilde{\nu}(z)+g(z))^{-1}
$$

is holomorphic in $V_{J}$.

REMARK. If $J_{1} \subset J_{2}$ are two intervals for which $\tau_{J_{1}}, \tau_{J_{2}}$ are defined, $\tau_{J_{1}}$ is the restriction to $J_{1}$ of $\tau_{J_{2}}$. Thus we may say that we have defined a positive measure $\tau=\tau(\nu, g)$ on $U \cap(\boldsymbol{R}-E)$. It is not hard to see that $\tau$ may be extended to a measure defined also in the neighborhood of a point $c \in E$ provided that

$$
g^{\prime}(c)>\frac{d \nu(t)}{(c-t)^{2}}
$$

for the extended measure $c$ is a negative point mass 


$$
\tau(\{c\})=-\left\{g^{\prime}(c)-\int \frac{d \nu(t)}{(c-t)^{2}}\right\}^{-1}
$$

In the special case in which $g(z)=-\sigma$ a real constant, the set $E$ is empty for any $\nu$. We denote by $T(\sigma) \nu=\tau(\nu, g)$ the measure given by Lemma 12. For $\sigma=0$ and $\nu$ the zero measure $T(\sigma) \nu$ is not defined; we exclude this case by supposing that $\nu$ is not the zero measure. In Appendix A we will use the measures $T(\sigma) \nu$ to remove the restriction in Lemma 11 to holomorphic functions $g(z)$.

Proof of Theorem 3. We are now ready to prove Theorem 3. It is convenient to have $0 \notin U$. If $0 \in U$, we begin by replacing $U$ by $U-\{0\}$.

Let $J$ be an interval whose closure is contained in $I-E$. Given an increasing sequence $\left\{D_{N}\right\}$ of finite dimensional subspaces of $\mathfrak{S}$, we can construct finite rank approximations $T_{N}(z)$ to $S(z)$ acting in $D_{N}$, which satisfy conditions (a) - (d) of the final paragraph of Theorem 3. To do this we first form $P_{N} S(z) P_{N}$, and use (1.1) to write it as the sum of the Hilbert transform of a measure on $J$ whose values are positive operators on $D_{N}$, and a function holomorphic on $J$ whose values are operators on $D_{N}$. Then to obtain $T_{N}(z)$ we replace the measure by a weak* approximation which is a finite sum of point masses, each a positive rank 1 operator.

Define

$$
\widetilde{T}_{N}(z)=T_{N}(z)\left\{1+\frac{z K T_{N}(z)}{1-z \operatorname{tr}\left(K T_{N}(z)\right)}\right\}
$$

for $z \in U$ such that $T_{N}(z)$ is defined, and $1-z \operatorname{tr}\left(K T_{N}(z)\right) \neq 0$.

The sets $\widetilde{U}, \widetilde{E}$ will be defined by specifying, for each $J$, the sets $\widetilde{U} \cap(U-(I-J)), \widetilde{E} \cap J$. It will be evident that this is done consistently. Write $K=|K| \operatorname{sgn} K$, where $|K|$ is positive and

$$
\operatorname{sgn} K= \pm 1 \text {. }
$$

Apply Lemma 10 to the positive measure $\omega(t)=\operatorname{tr}(|K| \nu(t))$ on $J$, and the function $g(z)=\operatorname{tr}\left(|K| R_{J}(z)\right)-z^{-1}(\operatorname{sgn} K)$, holomorphic in $U-(I-J)$. Since $\tilde{\omega}(z)+g(z)=z^{-1}(-1+z \operatorname{tr}[K S(z)])(\operatorname{sgn} K) \not \equiv 0$ we are not in the trivial case. Lemma 9 gives a certain discrete subset $E(\omega, g)$ of $U-(I-J)$, and we set

$$
\tilde{U} \cap(U-(I-J)=U-(1-J)-E(\omega, g),
$$

and $\widetilde{E} \cap J=E(\omega, g) \cap J$.

Next in Lemma 11 note that we could consider, instead of the single function $g(z)$, a sequence $g_{N}(z)$ of functions holomorphic in $U$ 
and converging to $g(z), \varphi_{N}(z)$ then being defined as $\left(\widetilde{\nu}_{N}(z)+g_{N}(z)\right)^{-1}$. The conclusion of the Lemma, and also the results established in the course of the proof are otherwise unchanged. We will refer to this trivial generalization of Lemma 11 as Lemma $11^{\prime}$.

Lemma $11^{\prime}$ is to be applied to the measures $\omega_{N}(t)=\operatorname{tr}\left(|K| \nu_{N}(t)\right)$, which converge to $\omega(t)$, and the functions

$$
g_{N}(z)=\operatorname{tr}\left(|K| P_{N} R_{J}(z) P_{N}\right)-z^{-1}(\operatorname{sgn} K),
$$

which converge to $g(z)$. The proof of the lemma shows that if $\widetilde{J}$ is an open interval whose closure is contained in $J$, and does not intersect $\widetilde{E}$, then $\widetilde{J}$ has a complex neighborhood $V_{\widetilde{J}}$ such that, for sufficiently large $N$,

$$
\left|\tilde{\omega}_{N}(z)+g_{N}(z)\right| \geqslant B|\operatorname{Im} z| \quad z \in V_{\widetilde{J}}
$$

for some $B>0$. Thus $\widetilde{T}_{N}(z)$ is defined for $z \in V_{\widetilde{J}}$ with $\operatorname{Im} z \neq 0$, as also is $\widetilde{S}(z)$. The proof of the lemma shows also that, for sufficiently large $N$, the poles of $\left(\tilde{\omega}_{N}(x)+g_{N}(x)\right)^{-1}$ on the real axis are simple with negative residues.

Let $f$ be any vector in $\mathscr{S}$. We wish to represent $(f, \widetilde{S}(z) f)=$ $\psi(z), z \in V_{\widetilde{J}}$, as the sum of the Hilbert transform of a positive measure on $\widetilde{J}$ (with the proper dependence on $f$ ) and a function holomorphic in $V_{\widetilde{J}}$. To do this we consider the functions

$$
\psi_{N}(z)=\left(f, \widetilde{T}_{N}(z) f\right),
$$

which are meromorphic in $V_{\widetilde{J}}$. A pole of $\psi_{N}(z)$ is either

( $\alpha$ ) a pole of $T_{N}(z)$

or

$$
\text { ( } \beta) \text { a zero of } 1-\mathrm{z} \operatorname{tr}\left(K T_{N}(z)\right) \text {. }
$$

Call the pole $z=z_{0}$. We compute the corresponding residue:

$(\alpha)$ let $R_{0}$ be the residue of $T_{N}(z)$ at $z=z_{0}$. Then, since $R_{0}$ and $K$ both have rank 1, we find that we must have $R_{0} K=0$ (otherwise, the pole does not persist in $\left.T_{N}(z)\right)$ and then the residue is $\left(f, R_{0} f\right) \geqslant 0$

( $\beta)$ The residue is

$$
\frac{\left(f, T_{N}\left(z_{0}\right) K T_{N}\left(z_{0}\right) f\right)}{-\operatorname{tr}\left(K T_{N}^{\prime}\left(z_{0}\right)\right)-z_{0}^{-2}}>0
$$

Thus the residues are positive. Note also that the formulae show that the residues of $\widehat{T}_{N}(z)$ are of rank 1 .

For $\operatorname{Im} z \neq 0, z \in V_{\widetilde{J}}$ we have

$$
|\operatorname{Im} z|^{3} \psi_{N}(z) \longrightarrow|\operatorname{Im} z|^{3} \psi(z) \quad \text { as } N \rightarrow \infty
$$


uniformly on compacts contained in $V$. To obtain the representation (1.1) for $\widetilde{S}(z), \widetilde{J}$, we have thus only to apply Lemma 9 to the functions $\psi_{N}(z)$.

It remains to show we can obtain an approximation theorem on the real axis for $S(z)$ if the approximations $T_{N}(z)$ satisfy (e) in addition to (a) - (d). For definiteness suppose $K$ is positive, so we can write $K=k \otimes k$ (if $K$ is negative we can write $K=-k \otimes k$, and make appropriate changes of sign). We compute

$\left(f, \widetilde{T}_{N}(z) \widetilde{K}(z) g\right)-(f, g)=\left\{\left(f, T_{N}(z) K(z) g\right)-(f, g)\right\}$

$$
\begin{aligned}
& +z\left(f, T_{N}(z) k\right)\left\{\frac{\left(k, T_{N}(z) K(z) g\right)-(k, g)}{\left\{1-z\left(k, T_{N}(z) k\right)\right\}}\right\} \\
& +z\left(f, T_{N}(z) k\right)\left(k-P_{N} k, g\right) \\
& +z^{2} \frac{\left(f, T_{N}(z) k\right)\left(k, T_{N}(z) k\right)\left(k-P_{N} k, g\right)}{\left\{1-z\left(k, T_{N}(z) k\right)\right\}} .
\end{aligned}
$$

By hypothesis the sequences $\left\{\left(f, T_{N}(z) K(z) g\right)-(f, g)\right\}$, and

$$
\left\{\left(k, T_{N}(z) K(z) g\right)-(k, g)\right\} \longrightarrow 0
$$

in measure $(\mu)$ as $N \rightarrow \infty$. The sequence $\left\{\left(k-P_{N} k, g\right)\right\} \rightarrow 0$ as $N \rightarrow \infty$. The remaining sequences $\left\{\left(f, T_{N}(z) k\right)\right\},\left\{\left(k, T_{N}(z) k\right)\right\},\left\{\left[1-z\left(k, T_{N}(z) k\right)\right]^{-1}\right\}$ which appear in (3.21) are bounded in measure $(\mu)$ as $N \rightarrow \infty$. Hence, by Lemma $7,(3.21) \rightarrow 0$ in measure $(\mu)$ as $N \rightarrow \infty$.

REMARK. In Theorem 1 we suppose $C(z)=C$ independent of $z$ so that $K(z)$ has the form

$$
K(z)=K-z(1+C) .
$$

If $(1+C)$ is invertible we may write

$$
T=(1+C)^{-1} K
$$

and define a new scalar product

$$
[x, y]=((1+C) x, y) .
$$

This product will generally not be positive definite since $1+C$ may have a finite number, $\chi$ (say), of negative eigenvalues. $\mathfrak{S}$ together with the product $[\cdot, \cdot]$ is then a Pontryagin space $\mathfrak{S}_{\chi}$ of index $\chi$, as defined in [11]. The operator $T$ is hermitian with respect to $[\cdot, \cdot]$, i.e., $[T x, y]=[x, T y]$ for all $x, y$. The representation (1.1) essentially gives a spectral representation for the resolvent of $T$; the existence of such a spectral representation for a self-adjoint operator in a Pontryagin space was established by Krein and Langer [10]: a slightly more general theorem is given by Langer [12]. To make the 
connection with [10] it is necessary to extend the measure $\nu$ across the points of $E$ when this is possible, as indicated in the remark following Lemma 12. In the terminology of [11] points of $E$ are critical points, regular if (3.11) is well-defined and singular otherwise.

4. A stronger form of Theorem 1. Theorem 1 is not applicable in the context to be described in $\S 5$. In this section we prove a strengthened version of Theorem 1 (Theorem 4). Theorem 4 is obtained from Theorem 1 by weakening certain of the conditions; for the reader's convenience we give a full statement, although this entails some repetition.

THEOREM 4. Let $\{K(z)\}$ be a family of bounded operators on $\mathfrak{S}$, defined and holomorphic in $z$, for $z$ in a complex neighborhood $U$ of some finite open interval I of the real axis. Suppose that

(a) $K(z)$ is hermitian for $z \in I$

(b) $K(z)=K_{1}(z)+D(z)$ for $z \in U$ where (b1) $D(z)$ is compact valued and holomorphic for $z \in U$.

(b2) For each $z \in I$ the pair of hermitian operators $K=K_{1}(z)$ and $L=-K_{1}^{\prime}(z)$ satisfy the following conditions (i), (ii) for some positive constants $A, B$ (possibly dependent on $z$ )

(i) $\|x\| \leqslant A\|K x\|+B\|L x\|$ for all $x \in \mathfrak{F}$

(ii) if $-d$ is the lower bound of the numerical range of $L$

$$
2 B^{2}\|L\| d<1
$$

(c) for some $z \in I, K(z)$ has nullity zero.

Then there exists a discrete set $E \subset I$ such that, for each open interval $J$ whose closure is contained in $I-E, K(z)$ has a bounded inverse $S(z)$ for $z \in N_{J}-J$ (where $N_{J}$ is a certain complex neighborhood of $J)$. In $N_{J}-J, S(z)$ admits a representation

$$
S(z)=\int_{J} \frac{d \nu(t)}{z-t}+R_{J}(z)
$$

where $\nu$ is a positive operator valued measure on $J$, and $R_{J}(z)$ is holomorphic in $N_{J}$.

Suppose that the condition (i) of (b2) is replaced by

$$
\|x\|^{2} \leqslant A|(x, K x)|+B|(x, L x)| \quad \text { for all } x \in \mathfrak{F} \text {. }
$$

Let $\left\{D_{N}\right\}$ be an increasing sequence of finite dimensional subspaces

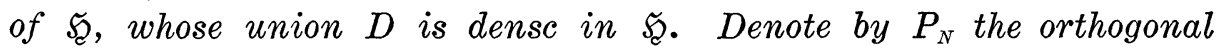
projection onto $D_{N}$. For sufficiently large $N$ the restriction of $P_{N} K(z) P_{N}$ to $D_{N}$ is invertible (as an operator on $D_{N}$ ) for all but a 
discrete set of $z \in I$ (dependent on $N$ ); when this inverse is defined denote by $T_{N}(z)$ its extension to an operator on $\mathfrak{S}$ which vanishes on the orthogonal complement of $D_{N}$. Let $f(z), g(z)$ be vector valued functions holomorphic in $z$ in a neighborhood of $I$. Then as $N \rightarrow \infty$

$$
\left(f(z), T_{N}(z) K(z) g(z)\right) \longrightarrow(f(z), g(z))
$$

in measure $(\mu)$ on $I$.

Complement to Theorem 4. We retain the notations and hypotheses of Theorem 4. Suppose that $h(z)$ is a vector valued function holomorphic in $z$ in a neighborhood of $I$, and that for each $x \in I-E$ the equation

$$
K(x) u=h(x)
$$

has a unique solution $u=g(x)$. Suppose that the function $x \rightarrow g(x)$ is weakly measurable on $I-E$, and that the function $x \rightarrow\|g(x)\|$ is Lebesgue integrable on any interval $J$ whose closure is contained in $I-E$. Then $g(x)$ can be extended to a function $g(z)$, defined and holomorphic on a neighborhood of $I-E$. In particular,

$$
\left(f(z), T_{N}(z) h(z)\right) \longrightarrow(f(z), g(z))
$$

in measure $(\mu)$ on $I$ as $N \rightarrow \infty$ by the second part of Theorem 4 .

REMARKs. If $K(z)$ satisfies condition (b) of Theorem 1 we may choose some $z_{0} \in I$ and set

$$
\begin{gathered}
D(z)=\int_{z_{0}}^{z} C(z) d z \\
K_{1}(z)=K\left(z_{0}\right)+\left(z-z_{0}\right) 1 .
\end{gathered}
$$

Then $D(z)$ satisfies condition (b1) of Theorem 4 , and $K_{1}(z)$ satisfies (b2) with $A=0, B=1$.

The rather clumsy conditions (i), (ii) are to be understood essentially as a positivity condition on $L$. If $L$ is strongly positive, $L \geqslant(-d) 1$ with $d<0$, and (i), (ii) hold with $A=0$ and $B=(-d)^{-1}$. If $L$ is positive, and $d \leqslant 0$ and (ii) holds for any $B$. (i) then requires $\|K x\|$ not to be small for those $x \in \mathfrak{S}$ for which $\|L x\|$ is small. In the approximation theorem we replace (i) by the stronger condition (i)' because it does not follow that if $K, L$ satisfy (i), (ii) then the approximations $K_{N}=P_{N} K P_{N}, L_{N}=P_{N} L P_{N}$ satisfy (i), (ii) (with the same constants $A, B$ ) as operators on $D_{N}$; for (i)', (ii) this is true. Note that (i), (ii) (or (i)', (ii)) are stable conditions in the sense that if $K, L$ satisfy them, and $K^{\prime}, L^{\prime}$ are sufficiently close to $K, L$ in 
norm, then $K^{\prime}, L^{\prime}$ satisfy them (with constants $A^{\prime}, B^{\prime}$ arbitrarily close to $A, B$ ). We may (and will) therefore suppose that the constants $A(z), B(z)$, which appear in (b2) (or (b2)') are continuous in $z$.

The problem of determining conditions on self-adjoint operators $K, L$ which will ensure that $K-z L$ has a bounded inverse for $z$ in some neighborhood of $z=0, \operatorname{Im} z \neq 0$, is closely related to the problem of determining conditions which will ensure that the commutator equation

$$
[K, M]=i L
$$

has no solution $M \in B(\mathfrak{F})$. For in the first problem $K$ may be supposed not to have a bounded inverse (otherwise it is trivial). But then for no $z$ can $K(z)=\exp [-i z M] K \exp [+i z M]$ be invertible; for this $K(z), K^{\prime}(0)=i[K, M]$. The commutator equation is discussed in Putnam [16] (\$2.10).

Condition (c) of Theorem 4 differs from the corresponding condition in Theorem 1 in that the point $z$ referred to in the condition is on the real axis. This change is essential for the application in $\S 5$. For the proof it is also essential since, the hypotheses of Theorem 4 do not imply that $K_{1}(z)$ is boundedly invertible for all $z \in U-I$, but only for $z \in V-I$, where $V$ is a smaller neighborhood of $I$.

The regularity conditions imposed on $g(x)$ in the complement to Theorem 4 are dictated by our proof and may possibly be unnecessary. In the proof of Theorem 4 it will be shown that if $x \in E, K(x)$ has a positive nullity, so the exclusion of $E$, from the set on which $K(x) u=h(x)$ is to have a unique solution, is essential; however, in general, even if $x \notin E, K(x)$ may have positive nullity. If $K(x)$ has positive nullity, then from (4.1) it follows that $x$ is a point of discontinuity for $\nu(t)$; this set of points is at most countable (if $K(x)=K-x 1$, it is the point spectrum of $K$ ).

Proof of Theorem 4. The proof of Theorem 4 is parallel to that of Theorem 1. First we prove Theorem 4 in the special case $D(z) \equiv 0$. In this case condition (c) is redundant. Then we use the perturbation result, Theorem 3, to pass to the general case. (For the approximation theorem it is necessary to generalize Theorem 3 by replacing the vectors $f, g$ which appear in the second part of Theorem 3 by the vector valued functions $f(z), g(z)$ which appear in the statement of Theorem 4; this is a trivial generalization and entails no change in the proof of $\S 3$ ).

Lemma 13. Theorem 4 is true if $D(z) \equiv 0$.

Proof. Without loss of generality suppose $0 \in I$. Write $K=K(0)$, 
$L=-K^{\prime}(0), A=A(0), B=B(0)$. If $L=0$, (i) requires $K$ to be strongly positive. Then for $J$ a sufficiently small neighborhood of 0 the conclusions of Theorem 4 hold trivially, since $K(z)$ is boundedly invertible for $z$ in a neighborhood of 0 . We therefore suppose $\|L\| \neq 0$.

Choose $d_{1} \geqslant d$ so that $\|L\| \geqslant d_{1}>0$ and $2 B^{2}\|L\| d_{1}<1$ (if $d>0$ take $d_{1}=d$; if $d \leqslant 0$ take $d_{1}$ sufficiently small and positive). Define a continuous real valued function $f(y)$ by

$$
\begin{array}{ll}
f(y)=y & \text { if } y \geqslant d_{1} \\
f(y)=d_{1} & \text { if } y \leqslant d_{1}
\end{array}
$$

and set $L_{1}=f(L) . \quad L_{1}$ is strongly positive so its positive square root $L_{1}^{1 / 2}$ is defined and boundedly invertible. From the factorization

$$
K-z L_{1}=L_{1}^{1 / 2}\left(L_{1}^{-1 / 2} K L_{1}^{-1 / 2}-z 1\right) L_{1}^{1 / 2}
$$

follows the existence of $S_{1}(z)=\left(K-z L_{1}\right)^{-1}$ as a bounded operator for $\operatorname{Im} z \neq 0$, and the representation

$$
S_{1}(z)=\int \frac{L_{1}^{-1 / 2} d \nu_{1}(t) L_{1}^{-1 / 2}}{z-t}
$$

where $\nu_{1}$ is the spectral measure of the self-adjoint operator $L_{1}^{-1 / 2} K L_{1}^{-1 / 2}$.

Let $\eta>0$, and set

$$
E(\eta)=\int_{-\eta_{1}}^{\eta_{1}} d \boldsymbol{\nu}_{1}(t) .
$$

Condition (i) gives for any $x \in \mathfrak{F}$

$$
\begin{aligned}
\left\|L_{1}^{-1 / 2} E(\eta) x\right\| & \leqslant A\left\|L_{1}^{1 / 2}\left(L_{1}^{-1 / 2} K L_{1}^{-1 / 2}\right) E(\eta) x\right\|+B\left\|L L_{1}^{-1 / 2} E(\eta) x\right\| \\
& \leqslant A\|L\|^{1 / 2} \eta\|x\|+B\|L\|^{1 / 2}\|x\| \cdot
\end{aligned}
$$

For any $\varepsilon>0$ we may thus choose $\eta$ sufficiently small that

$$
\begin{aligned}
\left\|L_{1}^{-1 / 2} E(\eta) L_{1}^{-1 / 2}\right\| & \leqslant\left\|L_{1}^{-1 / 2} E(\eta)\right\|^{2}\left(\text { since } E(\eta)^{2}=E(\eta)\right) \\
& \leqslant B^{2}\|L\|+\varepsilon .
\end{aligned}
$$

Then (4.3) gives an estimate

$$
\left\|S_{1}(z)\right\| \leqslant\left(B^{2}\|L\|+2 \varepsilon\right)|\operatorname{Im} z|^{-1}
$$

for $|\operatorname{Re} z|<\eta / 2,|\operatorname{Im} z|<\varepsilon d_{1} \eta / 2$.

For $|\boldsymbol{z}|$ sufficiently small,

$$
\left\|K(z)-\left(K-z L_{1}\right)\right\| \leqslant|z|\left(2 d_{1}+\varepsilon\right) .
$$

If $\varepsilon$ is chosen sufficiently small that 


$$
\left(2 d_{1}+\varepsilon\right)\left(B^{2}\|L\|+2 \varepsilon\right)<1
$$

(and this is possible by condition (ii)), (4.4) and (4.5) imply

$$
\left.\|\left[K(z)-K-z L_{1}\right)\right] S_{1}(z) \|<1
$$

for $z$ in some sector $R(0)$, with vertex 0 , of the form

$$
R(0)=\{z|| \operatorname{Im} z|<\tau,| \operatorname{Re} z|<\lambda| \operatorname{Im} z \mid\}
$$

with $\lambda>0, \tau>0$. Then

$$
S(z)=S_{1}(z)\left(1+\left[K(z)-\left(K-z L_{1}\right)\right] S_{1}(z)\right)^{-1}
$$

exists and is holomorphic in $R(0)$, and is the inverse of $K(z)$.

As noted in the remarks following the statement of Theorem 4 the constants $A(z), B(z)$ appearing in b2 can be supposed continuous in $z$. The preceding argument then shows that for any $z_{0} \in I, S(z)$ exists and is holomorphic in a sector $R\left(z_{0}\right)$ with vertex $z_{0}$ whose parameters $\lambda\left(z_{0}\right), \tau\left(z_{0}\right)$ vary continuously with $z_{0}$. For $J$ an open interval with closure contained in $I$, we define $N_{J}$ to be the union of the sectors $R\left(z_{0}\right)$ for $z_{0} \in J$.

To obtain the representation (4.1) of $S(z), z \in N_{J}-J$, we proceed as in the proof of Theorem 2. Let $\bar{D}=U \bar{D}_{N}$ be a domain dense in $\mathfrak{S}$, the finite dimensional subspaces $\bar{D}_{N}$ forming an increasing sequence (the superscript is introduced to distinguish these subspaces, which we are free to choose, from the subspaces $D_{N}$ given in the second half of the statement of Theorem 4). We seek to construct approximations $\bar{K}_{N}(z)$ to $K(z)$ such that

$(\alpha) \quad \bar{K}_{N}(z)$ is reduced by $\bar{D}_{N}$ and is zero on the orthogonal complement of $\bar{D}_{N}$

(ß) $\bar{K}_{N}(z) \rightarrow K(z)$ strongly as $N \rightarrow \infty$ for $z \in N_{J}$

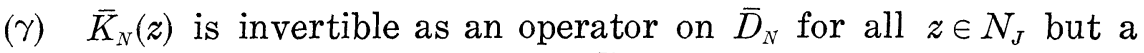
finite set of points on the real axis. $\bar{T}_{N}(z)$ the extension to $\mathfrak{S}$ of the inverse of $\bar{K}_{N}(z)$ is meromorphic in $N_{J}$, with simple poles on $J$ having positive residues.

(ס) for some integer $k,|\operatorname{Im} z|^{k} \bar{T}_{N}(z) \rightarrow|\operatorname{Im} z|^{k} S(z)$ strongly as $N \rightarrow \infty$, uniformly on compacts contained in $N_{J}$. Indeed, for $f(z)$, $g(z)$ holomorphic vector valued functions in $N_{J}$

$$
|\operatorname{Im} z|^{k}\left(f(z), \bar{T}_{N}(z) g(z)\right) \longrightarrow|\operatorname{Im} z|^{k}(f(z), S(z) g(z)) \quad \text { as } N \rightarrow \infty,
$$

uniformly on compacts contained in $N_{J}$.

The representation of $S(z)$ is then obtained from the representation of the approximations $\bar{T}_{N}(z)$ with the help of Lemma 9.

If $K(z)$ satisfies (b2)' we can simply take $\bar{K}_{N}(z)=\bar{P}_{N} K(z) \bar{P}_{N}$, for then $\bar{K}_{N}(z)$ satisfies (b2)' with the same constants $A(z), B(z)$, as $K(z)$. 
If $K(z)$ only satisfies the weaker condition (b2) this choice is not possible; in the next paragraphs we show how by replacing $N_{J}$ by a smaller neighborhood of $J$ and making a different choice of $\bar{K}_{N}(z)$ this difficulty can be circumvented.

Let $\left\{D_{N}\right\}$ be an increasing sequence of finite dimensional subspaces of $\mathfrak{S}$, whose union is dense in $\mathscr{S}$. Denote by $P_{N}$ the orthogonal projection onto $D_{N}$ and write $K_{N}(z)=P_{N} K(z) P_{N}$. For fixed $N$ and $x \in D_{N}$ with $\|x\|=1$ we have

$$
A(z)\left\|K_{M}(z) x\right\|+B(z)\left\|K_{M}^{\prime}(z) x\right\| \longrightarrow A(z)\|K(z) x\|+B(z)\left\|K^{\prime}(z) x\right\|
$$

as $M \rightarrow \infty$, uniformly in $x$ and in $z$, for $z$ in $J$. Choose

$$
B_{1}(z)=B(z)+\eta \text {, }
$$

with $\eta>0$ sufficiently small that for $z$ in $J$

$$
2 B_{1}(z)^{2}\left\|K^{\prime}(z)\right\| d(z)<1
$$

(where $d(z)$ is the upper bound of the numerical range of $K^{\prime}(z)$ ). Then for $M=M(N)$ sufficiently large

$$
\|x\| \leqslant(A(z)+1)\left\|K_{M}(z) x\right\|+B_{1}(z)\left\|K_{M}^{\prime}(z) x\right\|
$$

for all $x \in D_{N}, z \in J$. Now define $\bar{D}_{N}=D_{M(N)}$ and

$$
\bar{K}_{N}(z)=K_{M(N)}(z)+(1+H)\left(P_{M(N)}-P_{N}\right)
$$

where $H=\sup \|K(z)\|, z \in J$. We claim that for suitable $A^{\prime}(z), B^{\prime}(z)$ (independent of $N$ ) $\bar{K}_{N}(z)$ satisfies (b2) (as a family of operators on $\bar{D}_{N}$ ). Let $\varepsilon>0$. If $x \in \bar{D}_{N}$ is such that $\left\|x-P_{N} x\right\| \leqslant \varepsilon\|x\|,(4.6)$ gives $\|x\| \leqslant(1-\varepsilon)^{-1}\left\|P_{N} x\right\|$

$$
\begin{aligned}
\leqslant & (1-\varepsilon)^{-1}(A(z)+1)\left\|K_{M}(z) P_{N} x\right\|+(1-\varepsilon)^{-1} B_{1}(z)\left\|K_{M}^{\prime}(z) P_{N} x\right\| \\
\leqslant & (1-\varepsilon)^{-1}(A(z)+1)\left\|\bar{K}_{N}(z) x\right\|+(1-\varepsilon)^{-1} B_{1}(z)\left\|\bar{K}_{N}^{\prime}(z) x\right\| \\
& +\|x\|\left\{(A(z)+1)(2+H)+B_{1}(z)\left\|K^{\prime}(z)\right\|\right\} \varepsilon(1-\varepsilon)^{-1} .
\end{aligned}
$$

Choose $B^{\prime}(z)=B_{1}(z)+\delta$, with $\delta>0$ sufficiently small that for $z \in J$

$$
2 B^{\prime}(z)^{2}\left\|K^{\prime}(z)\right\| d(z)<1 \text {. }
$$

Then for $\varepsilon>0$ sufficiently small (4.7) gives

$$
\|x\| \leqslant(A(z)+2)\left\|\bar{K}_{N}(z) x\right\|+B^{\prime}(z)\left\|\bar{K}_{N}(z) x\right\| \cdot
$$

If $x \in \bar{D}_{N}$ is such that $\left\|x-P_{N} x\right\| \geqslant \varepsilon\|x\|$

$$
\|x\| \leqslant \varepsilon^{-1}\left\|x-P_{N} x\right\| \leqslant \varepsilon^{-1}\left\|\bar{K}_{N}(z) x\right\|
$$

since $\bar{K}_{N}(z) \geqslant 1$ on the orthogonal complement of $D_{N}$ in $\bar{D}_{N}$. Thus if we set $A^{\prime}(z)=\max \left[\varepsilon^{-1}, A(z)+2\right],(4.8),(4.9)$ imply that b2 (i) 
holds for $\bar{K}_{N}(z)$ on $\bar{D}_{N}$ with constants $A^{\prime}(z), B^{\prime}(z)$, and $z \in J$. Condition b2 (ii) holds by virtue of the choice of $B^{\prime}(z)$ and the fact that the numerical range of $\bar{K}_{N}^{\prime}(z)$ is contained in that of $K^{\prime}(z)$.

It remains to check that the $\bar{K}_{N}(z)$ satisfy the desired conditions $\alpha, \beta, \gamma$, and $\delta . \quad \alpha, \beta$ are clear from the definition of $\bar{K}_{N}(z) . \gamma$ is proved by the argument used to show that $S(z)=[K(z)]^{-1}$ exists and is holomorphic in $N_{J}$; it is only necessary to replace $K(z)$, $\mathfrak{S}$ by $\bar{K}_{N}(z)$, $D_{N}$. The statements regarding the behavior of $\bar{T}_{N}(z)=\left[\bar{K}_{N}(z)\right]^{-1}$ on $J$ follow from the spectral theorem in a finite dimensional space. Finally $\delta$ is proved following the pattern of the proofs of Lemmas 1, 2.

For the approximation theorem (the second part of Theorem 4) we insist on the stronger condition (b2)'. Then in the above argument we can simply take $\bar{K}_{N}(z)=K_{N}(z)$, and the approximation conditions $\alpha, \beta, \gamma$, and $\delta$ hold as before. The proof of the approximation theorem is then completed by an application of Lemma 6 (compare the corresponding argument in the proof of Theorem 2 , which follows Lemma 8).

Proof of Theorem 4 (continued). Let $z_{0}$ be the point of $I$ such that $K\left(z_{0}\right)$ has nullity zero, whose existence is guaranteed by condition (c). In view of the stability of conditions (i), (ii) noted in the Remark following the statement of Theorem 4, we may choose $F, G$ symmetric operators of finite rank so that

$$
K_{1}(z)+(D(z)-[G-z F])=K_{2}(z)
$$

satisfies b2 for $z$ in a sufficiently small neighborhood of $z_{0}, U\left(z_{0}\right)$ (say). Denote by $V$ a finite dimensional subspace of $\mathfrak{S}$ containing the ranges of $F, G$ and by $W$ the image under $K\left(z_{0}\right)^{-1}$ (the possibly unbounded inverse of $K\left(z_{0}\right)$ ) of the intersection of $V$ and the range of $K\left(z_{0}\right)$. Denote by $X$ the vector space of pairs of symmetric operators $\sigma=\left(\sigma_{1}, \sigma_{2}\right)$ with ranges contained in $V$. For $\sigma \in X$ write

$$
K_{\sigma}(z)=K_{2}(z)+\left(\sigma_{1}-z \sigma_{2}\right),
$$

and define $P \subset X$ by

$$
\begin{aligned}
P= & \left\{\sigma \mid \text { Theorem } 4 \text { holds for } K_{\sigma}(z) \text { in } U\left(z_{0}\right)\right. \text { with an exceptional } \\
& \text { set } \left.E_{\sigma} \text { not containing } z_{0}\right\} .
\end{aligned}
$$

By Lemma $13(0,0) \in P$; we will prove $(G, F) \in P$.

For $\sigma \in X$ denote by $r(\sigma)$ the sum of the ranks of $\sigma_{1}, \sigma_{2}$.

Lemma 14. If $\sigma \in P$ and $\tau \in X$ with $r(\tau)=1$ then

(I) $\sigma+\lambda \tau \notin P$ for at most one real $\lambda$

(II) if $\sigma+\lambda \tau=\sigma^{\prime} \notin P$ then $K_{\sigma^{\prime}}\left(z_{0}\right)$ has a nonzero null vector. 
Conclusion of proof of Theorem 4 (granting Lemma 14). Let $(G, F)=\sum_{i=1}^{m} \tau_{i}$ be a decomposition of $(G, F)$ with $r\left(\tau_{i}\right)=1$ for all $i$. By induction from Lemma 14 (I) there exist real numbers $\lambda_{i}$ which may be chosen arbitrarily small such that $\sum_{i=1}^{m}\left(1+\lambda_{i}\right) \tau_{i} \in P$. If $(G, F) \notin P$, Lemma 14 (II) then implies that for some integer $k$, $1 \leqslant k<m, \sum_{i=1}^{k} \tau_{i}+\sum_{i=k+1}^{m}\left(1+\lambda_{i}\right) \tau_{i}=\sigma^{\prime}$ is such that $K_{\sigma^{\prime}}\left(z_{0}\right)$ has a nonzero null vector. By choice of the $\lambda_{i}, \sigma^{\prime}$ may be made arbitrarily close to $(G, F)$. Let $v^{\prime}$ be a null vector for $K_{\sigma^{\prime}}\left(z_{0}\right)$ of unit length. Then

$$
K\left(z_{0}\right) v^{\prime}=\left[\left(G-z_{0} F\right)-\left(\sigma_{1}^{\prime}-z_{0} \sigma_{2}^{\prime}\right)\right] v^{\prime} \in V,
$$

so $v^{\prime} \in W$. Moreover, $\left\|K\left(z_{0}\right) v^{\prime}\right\|$ may be made arbitrarily small by choice of $\sigma^{\prime}$. Since the unit ball of $W$ (which is finite dimensional) is compact, we may construct a sequence of unit vectors $v_{n}^{\prime} \in W$ convergent to some vector $v$, and such that $\lim _{n \rightarrow \infty}\left\|K\left(z_{0}\right) v_{n}^{\prime}\right\|=0$. But then $v^{\prime}$ is a nonzero null vector of $K\left(z_{0}\right)$. This contradiction shows $(G, F) \in P$, i.e., that Theorem 4 holds for $K(z)$ in $U\left(z_{0}\right)$.

It remains only to make the extension to the whole of $I$.

Let $I\left(z_{0}\right)$ be a maximal open interval containing $z_{0}$ and contained in $I$ such that the conclusions of Theorem 4 are valid for $I\left(z_{0}\right)$. If $I\left(z_{0}\right) \neq I$ then $I\left(z_{0}\right)$ has an end point $z_{1}$ (say) in $I$. Choose $G_{1}, F_{1}$ symmetric operators of finite rank so that

$$
K_{1}(z)+\left(D(z)-\left[G_{1}-z F_{1}\right]\right)=K_{3}(z)
$$

satisfies b2 for $z$ in a sufficiently small neighborhood of $z_{1}, I\left(z_{1}\right)$ (say). Lemma 13 shows that $K_{3}(z)$ is boundedly invertible for $\operatorname{Im} z \neq 0$ in a complex neighborhood of $I\left(z_{1}\right)$. This neighborhood will contain a point $z_{2}$ for which $K(z)$ is boundedly invertible (since $K(z)$ is boundedly invertible in a complex neighborhood of $I\left(z_{0}\right)$ minus a discrete set). It is therefore possible to use Theorem 3 as in the proof of Theorem 1 (cf. the argument following the statement of Theorem 3) to obtain the conclusions of Theorem 4 for $I\left(z_{1}\right)$. But this contradicts the maximality of $I\left(z_{0}\right)$. Hence $I\left(z_{0}\right)=I$.

Proof of Lemma 14. Since $r(\tau)=1$ one of $\tau_{1}, \tau_{2}$ is of rank 1 and the other of rank 0 . Suppose for definiteness that $\tau_{1}=0$ (the proof in case $\tau_{2}=0$ is essentially the same). Without loss of generality we may suppose $\tau_{1}$ positive so that $\tau_{1}=k \otimes k$ for some $k \in \mathfrak{K}$. Since $\sigma \in P$ we have for some open interval $J$ of $z_{0}$ a representation of $S_{o}(z)=\left[K_{\sigma}(z)\right]^{-1}$

$$
S_{\sigma}(z)=\int_{J} \frac{d \nu(t)}{z-t}+R(z)
$$

valid for $\operatorname{Im} z \neq 0$ in a complex neighborhood of $J$. Then Theorem 3 
shows that $\sigma^{\prime}=\sigma+\lambda \tau \in P$ unless

$$
1-\lambda z_{0}\left\{\int \frac{(k, d \nu(t) k)}{z_{0}-t}+\left(k, R\left(z_{0}\right) k\right)\right\}=0
$$

and

$$
z_{0}^{2}|\lambda|\left\{\int \frac{(k, d \nu(t) k)}{\left(z_{0}-t\right)^{2}}-\left(k, R^{\prime}\left(z_{0}\right) k\right)\right\} \leqslant \operatorname{sgn} \lambda .
$$

(Recall that in the proof of Theorem 3 given at the end of $\S 3$, the exceptional set $E$ appears as the set $E(\omega, g)$ of Lemma 10 for $\omega$ the positive measure trace $(|k| \nu(t))$ and $g$ the holomorphic function $g(z)=$ trace $(|K| R(z))-z^{-1}(\operatorname{sgn} K)$. Here $K=\lambda k \otimes k$.)

(I) follows from the linearity of (4.9) in $\lambda$.

To prove (II) we must construct a nonzero null vector $v$ for $K_{\sigma^{\prime}}\left(z_{0}\right)$ assuming (4.10), (4.11) hold.

Consider the antilinear functional on $\mathfrak{F}$ defined by

$$
V(y)=\int \frac{(y, d \nu(t) k)}{z_{0}-t}+\left(y, R\left(z_{0}\right) k\right) .
$$

(4.10) implies $V(k) \neq 0$ so $V$ is not identically zero. Since

$$
\begin{aligned}
\left|\int \frac{(y, d \nu(t) k)}{z_{0}-t}\right|^{2} & \leqslant\left\{\int \frac{(k, d \nu(t) k)}{\left(z_{0}-t\right)^{2}}\right\}\left\{\int(y, d \nu(t) y)\right\} \\
& \leqslant M\|y\|^{2}
\end{aligned}
$$

(using the positivity of the operator valued measure $\nu$, and (4.11)) $\mathrm{V}$ is everywhere defined and bounded, and so, by the Riesz representation theorem, can be written in the form $V(y)=(y, v)$, for a uniquely determined $v \in \mathfrak{S} . \quad v \neq 0$ since $V$ is not identically zero.

To prove $K_{\sigma^{\prime}}\left(z_{0}\right) v=0$, we must show that, for any $w \in \mathfrak{S}$,

$$
V\left(K_{\sigma^{\prime}}\left(z_{0}\right) w\right)=0
$$

$\left(K_{\sigma^{\prime}}\left(z_{0}\right)\right.$ is hermitian). Now

$$
\begin{aligned}
V\left(K_{\sigma^{\prime}}\left(z_{0}\right) w\right) & =\int \frac{\left(K_{\sigma^{\prime}}\left(z_{0}\right) w, d \nu(t) k\right)}{z_{0}-t}+\left(K_{\sigma^{\prime}}\left(z_{0}\right) w, R\left(z_{0}\right) k\right) \\
& =\lim _{\varepsilon \rightarrow 0+}\left\{\int \frac{\left(K_{\sigma^{\prime}}(z) w, d \nu(t) k\right)}{z-t}+\left(K_{\sigma^{\prime}}(z) w, R(z) k\right)\right. \\
& =\lim _{\varepsilon \rightarrow 0+}\left(\left[K_{\sigma}(z)-\lambda z k \otimes k\right] w, S_{\sigma}(z) k\right) \quad\left(\text { with } z=z_{0}+i \varepsilon\right) \\
& =\lim _{\varepsilon \rightarrow 0+}(w, k)\left(1-\lambda z\left(k, S_{\sigma}(z) k\right)\right) \\
& =0 \quad \text { by }(4.10) .
\end{aligned}
$$


Proof of the complement to Theorem 4. By Theorem $4 K(z)$ has a bounded inverse $S(z)$ for points $z$ with $\operatorname{Im} z \neq 0$ in some complex neighborhood $N_{J}$ of $J$, and $S(z)$ has a representation (4.1). For $z \in N_{J}-J$ define $g(z)=S(z) h(z) . g(z)$ is now defined for $z \in N_{J}$, and holomorphic for $z \in N_{J}-J$. To prove $g(z)$ is holomorphic in $N_{J}$, we will use the edge of the wedge theorem ([19], Theorem 2.16). According to that theorem it suffices to show that, for any $C^{\infty}$ function $\varphi(x)$ with support contained in $J$, and any vector $w \in \mathfrak{S}$

$$
\lim _{\varepsilon \rightarrow 0} \int \varphi(x)(w, g(x+i \varepsilon)) d x=\int \varphi(x)(w, g(x)) d x .
$$

Now

$$
\begin{aligned}
& \int \varphi(x)\{(w, g(x+i \varepsilon))-(w, g(x))\} d x \\
(4.13)= & \int \varphi(x)\{(w, S(x+i \varepsilon) h(x+i \varepsilon))-(w, S(x+i \varepsilon) K(x+i \varepsilon) g(x)) d x \\
= & \int \varphi(x)(w, S(x+i \varepsilon) 1(x, \varepsilon)) d x
\end{aligned}
$$

where

$$
1(x, \varepsilon)=(h(x+i \varepsilon)-h(x))-(K(x+i \varepsilon)-K(x)) g(x) .
$$

In (4.13) we substitute the representation (4.1) of $S(x+i \varepsilon)$. The term containing $R(x+i \varepsilon)$ converges to zero as $\varepsilon \rightarrow 0$ since the integral over $J$ of $\|1(x, \varepsilon)\|$ converges to zero. It ramains to consider

$$
\int \varphi(x)\left\{\int \frac{(w, d \nu(t) 1(x, \varepsilon))}{x+i \varepsilon-t}\right\} d x .
$$

Suppose, for the moment, that $g(x)$ satisfies a Lipschitz condition

$$
\left\|g\left(x_{1}\right)-g\left(x_{2}\right)\right\| \leqslant M\left|x_{1}-x_{2}\right|^{\alpha}, \quad \alpha>\frac{1}{2} .
$$

We expand $1(x, \varepsilon)$ in a Fourier series on the interval $J$

$$
1(x, \varepsilon)=\sum_{n=-\infty}^{+\infty} 1_{n}(\varepsilon) e_{n}(x)
$$

with $1_{n}(\varepsilon) \in \mathscr{S}$ and $\left\{e_{n}(x)\right\}$ the appropriate complex exponentials. By a theorem of Bernstein ([2], p. 154) the Lipschitz condition on $g(x)$ implies that its Fourier series converges absolutely; more precisely, for some $\gamma>0$, the Fourier coefficients $g_{n}$ of $g(x)$ satisfy

$$
\sum_{n=-\infty}^{+\infty}\left\|g_{n}\right\|\left(|n|^{r}+1\right)<\infty
$$

Since $h(x)$ and $K(x)$ are holomorphic in $x,(4.14)$ then shows that (4.16) 
converges absolutely and, for some $B>0$,

$$
\sum_{n=-\infty}^{+\infty}\left\|1_{n}(\varepsilon)\right\|\left(|n|^{\gamma}+1\right) \leqslant B \varepsilon .
$$

Substituting (4.16) into (4.15) we obtain a series whose $n$th term is

$$
\int \varphi(x)\left\{\int \frac{\left(w, d \nu(t) 1_{n}(\varepsilon)\right)}{x+i \varepsilon-t}\right\} e_{n}(x) d x .
$$

Now if $\psi(x)$ is a sufficiently smooth function of compact support we have the estimate

$$
\sup _{t}\left|\int \frac{\psi(x) d x}{x+i \varepsilon-t}\right| \leqslant K(\gamma)\|\psi\|_{r}
$$

where

$$
\|\psi\|_{\gamma}=\sup _{x}|\psi(x)|+\sup _{x, h}|h|^{-\gamma}|\psi(x+h)-\psi(x)|
$$

([6] p. 2419). We interchange the order of integrations in (4.18) and make use of (4.19) with $\psi_{n}(x)=\varphi(x) e_{n}(x)$ to obtain for (4.18) the bound

$$
K(\gamma)\left\|\psi_{n}\right\|_{\gamma}\|w\|\left\|1_{n}(\varepsilon)\right\|\|\nu(J)\| \leqslant A\left\|1_{n}(\varepsilon)\right\|\left(|n|^{r}+1\right)
$$

(4.17) then gives a bound $A B \varepsilon$ for (4.15), and this completes the proof that (4.13) converges to zero as $\varepsilon \rightarrow 0$.

If $g(x)$ is merely measurable with $\|g(x)\|$ integrable on $J$, we choose $\eta>0$ and make a decomposition

$$
g(x)=g_{1}(x)+g_{2}(x)
$$

with $g_{1}(x) \in \operatorname{Lip} \alpha(\alpha>1 / 2)$, and the $L^{1}$ norm of $\left\|g_{2}(x)\right\|$ less than $\eta$. (To obtain such a decomposition take $g_{1}=g * \delta$, with $\delta$ a smooth and sufficiently good approximation to the $\delta$ function.) The contribution of $g_{2}$ in (4.15) is bounded by

$$
\int \varphi(x) \frac{1}{\varepsilon}\|w\|\|K(x+i \varepsilon)-K(x)\|\left\|g_{2}(x)\right\|\|\nu(J)\| d x \leqslant C \eta
$$

(for some $C$ independent of $\varepsilon$ and $\eta$ ). The contribution of $g_{1}$ has limit 0 as $\varepsilon \rightarrow 0$ by the preceding argument so we conclude that the lim sup as $\varepsilon \rightarrow 0$ of the absolute value of (4.15) is $\leqslant C \eta$, $\eta$ was arbitrary so the convergence to zero of (4.15), and hence of (4.13), as $\varepsilon \rightarrow 0$ is proved.

REMARK. The argument used to handle the $g_{1}(x)$ term in the above proof is unnecessarily refined, for we can arrange to have 
$g_{1}(x)$ as smooth as we wish. However, the appearance of the Lip $\alpha$ $\alpha>1 / 2$ condition is interesting. The limiting values as $\varepsilon \rightarrow 0$ of integrals of the type (4.15) have been studied by Brownell [3], [4]; the same Lipschitz condition appears in Lemma $3 \mathrm{~b}$ of [4]. It seems likely then that the Fourier approximation used here could be used to give an alternative proof of Brownell's results.

5. The Kohn variational method. Let $v(r)$ be a real valued function defined on $[0, \infty), E$ a real number $>0$. The differential equation with boundary condition

$$
\left(\frac{-d^{2}}{d r^{2}}+v(r)-E\right) w=0, \quad w(0)=0
$$

has, for $v(r)$ satisfying suitable regularity conditions (in particular decreasing sufficiently rapidly as $r \rightarrow \infty$ ), a unique complex valued solution of the form

$$
w_{s}(r)=\exp (i k r)-\lambda \exp (-i k r)+\varphi(r) .
$$

In (5.2) $k=\sqrt{E}, \lambda$ is a complex number and $\varphi \in L^{2}[0, \infty)$. Since the complex conjugate of $w_{s}(r)$ also satisfies (5.1) and has the form (5.2), it follows from the uniqueness that $\lambda$ has modulus 1 .

(5.1) appears in quantum mechanics as a reduced form of the Schroedinger equation for a particle moving with energy $E$ and zero angular momentum in a central field of force characterized by a reduced potential $v(r)$ ([8], p. 246). The solution (5.2) describes the scattering of the particle by the potential; $\lambda$ determines the $s$-wave phase shift $\delta, \lambda=\exp (-2 i \delta)$.

Note that $\varphi(r)$ will not satisfy the boundary condition at $r=0$. It is convenient to rewrite (5.2) in a form in which all the functions which appear satisfy the boundary condition at $r=0$. Choose $\alpha>0$ and set

Then

$$
\begin{aligned}
& w_{+}(r)=\exp (i k r)(1-\exp (-\alpha r)) \\
& w_{-}(r)=\exp (-i k r)(1-\exp (-\alpha r)) .
\end{aligned}
$$

$$
w_{s}(r)=w_{+}(r)-\lambda w_{-}(r)+\psi(r)
$$

and again $\lambda \in C$ and $\psi \in L^{2}[0, \infty)$ are uniquely determined by the requirement that $w_{s}(r)$ satisfy (5.1).

Nuttall [14] has shown how to formulate the problem of determining $\lambda$ and $\psi$ in the language of Hilbert space. Substitution of (5.3) into (5.1) gives the equation

$$
\left.\left(-\frac{d^{2}}{d r^{2}}+v(r)-E\right)\right) \psi(r)=\psi_{+}(r)-\lambda \psi_{-}(r)
$$


where

$$
\begin{aligned}
& \psi_{+}(r)=v(r) w_{+}(r)+\left[2 i \alpha k-\alpha^{2}\right] \exp [i(k-i \alpha) r] \\
& \psi_{-}(r)=v(r) w_{-}(r)+\left[-2 i \alpha k-\alpha^{2}\right] \exp [-i(k+i \alpha) r]
\end{aligned}
$$

If $v(r) \in L^{2}[0, \infty), \psi_{+}(r)$ and $\psi_{-}(r)$ are in the Hilbert space $\mathfrak{S}=$ $L^{2}[0, \infty)$. The differential operator with the boundary condition may be reexpressed as a self-adjoint operator on $\mathfrak{S}$. In this way (5.4) gives

$$
\left(H_{0}+V-E\right) \psi=\psi_{+}-\lambda \psi_{-}
$$

where $H_{0}$ is the self-adjoint operator defined by $D\left(H_{0}\right)=\{w \mid w(0)=0$, $w$ is continuously differentiable, $(d w / d r)$ is absolutely continuous and $\left.\left(d^{2} w / d r^{2}\right) \in L^{2}[0, \infty)\right\}, H_{0} w=-\left(d^{2} w / d r^{-2}\right)$ if $w \in D\left(H_{0}\right)$ and $V$ the operator of multiplication by $v(r)$. Under suitable conditions on $v(r), H_{0}+V$ is self-adjoint with $D\left(H_{0}+V\right)=D\left(H_{0}\right)([9])$. A second Hilbert space equation may be obtained from (5.4) by multiplying (5.4) by $\bar{w}_{-}(r)$ and integrating over $[0, \infty)$. The term on the left of the resulting equation is finite and equal to $\left(\psi_{-}, \psi^{2}\right)$ provided that

$$
\lim _{r \rightarrow \infty}\left[\bar{w}_{-}(r) \psi^{\prime}(r)-\bar{w}_{-}^{\prime}(r) \psi(r)\right]=0
$$

and the terms on the right are finite if $v(r) \in L^{1}[0, \infty)$; the equation is

$$
\left(\psi_{-}, \psi\right)=p-\lambda q
$$

where

$$
\begin{aligned}
& p=\int_{0}^{\infty} \bar{w}_{-}(r) \psi_{+}(r) d r \\
& q=\int_{0}^{\infty} \bar{w}_{-}(r) \psi_{-}(r) d r .
\end{aligned}
$$

Note that (5.7) is not a consequence of (5.5), but is to be regarded as a Hilbert space formulation of the boundary condition (5.6) at infinity.

By choosing $\alpha$ sufficiently large, we can arrange to have $q \neq 0$. Then (5.7) can be solved for $\lambda$, and substitution for $\lambda$ in (5.5) gives

$$
\left[H_{0}+V-E-q^{-1} \psi_{-} \otimes \psi_{-}\right] \psi=\psi_{+}-p q^{-1} \psi_{-} \cdot
$$

The Kohn variational method ([8], p. 313 et. seq.) is an approximation procedure for the determination of $\lambda, \psi$. A sequence of linearly independent functions $\left\{\psi_{n}\right\}$ is chosen from $D\left(H_{0}\right)$ (the trial functions). This determines an increasing sequence of finite dimensional subspaces $D_{n}=\operatorname{span}\left\{\psi_{1}, \cdots, \psi_{n}\right\}$ of $\mathfrak{S}$. Denote by $P_{n}$ the orthogonal projector onto $D_{n}$. Then (5.8) is approximated by

$$
P_{n}\left[H_{0}+V-E-q^{-1} \psi_{-} \otimes \psi_{-}\right] P_{n} \psi_{n}=P_{n}\left[\psi_{+}-p q^{-1} \psi_{-}\right] \text {. }
$$


If the operator on the left of (5.9) is invertible on $D_{n}$, so that (5.9) has a unique solution $\psi_{n}$, the corresponding approximation $\lambda_{n}$ to $\lambda$ is given by

$$
\left(\psi_{-}, \psi_{n}\right)=p-\lambda_{n} q
$$

For a given energy $E$ there is little hope of proving that $\lambda_{n} \rightarrow \lambda$ as $n \rightarrow \infty$, since one cannot even be sure that the $\lambda_{n}$ are defined. However, Nuttall [14] was able to show (under suitable conditions on $v(r)$, and on the trial functions) that, on any finite interval $I \subset(0, \infty), \lambda_{n}(E) \rightarrow \lambda(E)$ in measure as $n \rightarrow \infty$. Nuttall pointed out that this result was not entirely satisfactory since it does not provide a justification for the use of the Kohn method to calculate the phase shift at a particular value of the energy. In that case the standard procedure is to vary the trial functions, thereby introducing a parameter $z$ which enters only into the approximations, not the physical quantity to be calculated. The resulting Kohn approximations may be expected to converge in measure in $z$. The problem of justifying this expectation was attacked by McCartor [13], who obtained some partial results. He obtained essentially Theorem 2, under an additional condition on the trial functions. The present work is a development of [13], [14], and gives a convergence theorem of the desired kind (Theorem 5 below).

It is convenient to rewrite (5.8) in a form in which unbounded operators do not appear. Let $c>0$. Then $R_{0}(c)^{1 / 2}=\left(H_{0}+c\right)^{-1 / 2}$ is a positive bounded operator. (5.8) gives

$$
\begin{aligned}
& \left\{1-(E+c) R_{0}(c)+R_{0}(c)^{1 / 2} V R_{0}(c)^{1 / 2}-q^{-1} R_{0}(c)^{1 / 2} \psi_{-} \otimes R_{0}(c)^{1 / 2} \psi_{-}\right\} \xi \\
= & R_{0}(c)^{1 / 2} \psi_{+}-p q^{-1} R_{0}(c)^{1 / 2} \psi_{-}
\end{aligned}
$$

where $\xi=\left(H_{0}+c\right)^{1 / 2} \psi$. $\lambda$ is given in terms of $\xi$ by

$$
\left(\psi_{-}, R_{0}(c)^{1 / 2} \xi\right)=p-\lambda q \text {. }
$$

The trial functions $\psi_{n}$ are transformed at the same time into

$$
\xi_{n}=\left(H_{0}+c\right)^{1 / 2} \psi_{n} \text {. }
$$

From now on $D_{n}$ will denote the span of $\left\{\xi_{1}, \cdots, \xi_{n}\right\}, P_{n}$ the projector onto $D_{n}$.

Following McCartor [13] we suppose that the parameter $z$ is introduced into the trial functions in such a way that

$$
P_{n}(z)=U(z)^{-1} P_{n} U(z)
$$

where $U(z)$ is, for $z$ real, a unitary operator. Then the problem can be transformed into one in which the trial functions are kept fixed and $z$ appears in the operator - namely 


$$
\begin{gathered}
U(z)\{\cdots\} U(z)^{-1} \xi(z)=U(z)\left\{R_{0}(c)^{1 / 2} \psi_{+}-p q^{-1} R_{0}(c)^{1 / 2} \psi_{-}\right\} \\
\left(U(z) R_{0}(c)^{1 / 2} \psi_{-}, \xi(z)\right)=p-\lambda q .
\end{gathered}
$$

In order that Theorem 4 should give a convergence theorem for the Kohn approximations $\lambda_{n}$ for $\lambda$ corresponding to (5.14), (5.15) and the sequence of trial functions $\xi_{n}$, it is necessary that the operators and vectors which appear in (5.14), (5.15) should admit holomorphic extensions for $z$ in some complex neighborhood of an interval of the real axis.

A simple choice for the $U(z)$ are the unitary operators induced by dilatations of $[0, \infty)$. Define

$$
(U(z) w)(r)=z^{1 / 2} w(z r)
$$

for $z$ real and positive, and $w \in \mathfrak{S}$. Then $U(z)$ is unitary. Using these transformations to vary the trial functions we obtain

THEOREM 5. Consider the s-wave scattering of a particle in a central potential. With the notations introduced above suppose

(i) that the span $D$ of the trial functions $\xi_{n}$ is dense in $\mathfrak{F}$

(ii) that the trial functions are varied in accordance with (5.13), the transformations $U(z)$ being given by (5.16)

(iii) that the reduced potential $v(r)$ is in $L^{1}[0, \infty)$, and of short range, i.e., for some $C>0, v(r) \exp (C r) \in L^{2}[0, \infty)$

(iv) that $v(r)$ is dilatation analytic in the sense that the operator $U(z) R_{0}(c)^{1 / 2} V R_{0}(c)^{1 / 2} U(z)^{-1}$ admits a holomorphic extension to some complex neighborhood of $z=1$

(v) that at the energy $E>0$ under consideration the equation (5.11), which determines the s-wave phase shift, has a unique solution.

Then the Kohn approximations $\lambda_{n}(z)$ to $\lambda$ converge in measure to $\lambda$ on any bounded subinterval of $(0, \infty)$.

REMARK. Condition (i) can also be expressed by saying that the $\psi_{n}=\left(H_{0}+c\right)^{-1 / 2} \xi_{n}$ should have a span which is a core for $H_{0}$ as a quadratic form (cf. [17]). Note that the Kohn approximations which we study in Theorem 5 after replacing (5.8) by (5.11) are not the same as those which are obtained from (5.10). The conclusion of Theorem 5 holds also for the approximations given by (5.10). To prove this one must consider in Theorem 4 nonorthogonal projectors $P_{N}, T_{N}(z)$ now being defined as the inverse of $P_{N}^{*} K(z) P_{N}$ on $D_{N}$. Approximations constructed using nonorthogonal projectors are considered also in [7]. For the use of condition (iv) cf. also [1].

Proof of Theorem 5. We claim that Theorem 5 follows from 
Theorem 4, and the complement to Theorem 4. First we list the identifications to be made, and then show that the conditions of Theorem 4 are satisfied

$$
\begin{aligned}
K_{1}(z) & =U(z)\left\{1-(E+c) R_{0}(c)\right\} U(z)^{-1} \\
D(z) & =U(z)\left\{R_{0}(c)^{1 / 2} V R_{0}(c)^{1 / 2}-q^{-1} R_{0}(c)^{1 / 2} \psi_{-} \otimes R_{0}(c)^{1 / 2} \psi_{-}\right\} U(z)^{-1} \\
h(z) & =U(z)\left\{R_{0}(c)^{1 / 2} \psi_{+}-p q^{-1} R_{0}(c)^{1 / 2} \psi_{-}\right\} \\
g(z) & =\xi(z) \\
f(z) & =U(z) R_{0}(c)^{1 / 2} \psi_{-} .
\end{aligned}
$$

The existence of an extension of $K_{1}(z)$ to complex $z$ follows from

$$
U(z) H_{0} U(z)^{-1}=z^{-2} H_{0}
$$

so

$$
K_{1}(z)=1-(E+c) z^{2} R_{0}\left(z^{2} c\right) .
$$

Conditions (iii) and (iv) of Theorem 5 give holomorphic extensions of $D(z), h(z)$, and $f(z)$ to a complex neighborhood of the real axis. $R_{0}(c)^{1 / 2} V R_{0}(c)^{1 / 2}$ is compact under the conditions imposed on $v(r)$; this is well-known (cf. [17], Theorem I.22). That $D(z)$ is compact valued follows.

Condition (c) of Theorem 4 is guaranteed by condition (v) of Theorem 5, as also is the uniqueness of $g(x)$ required for the complement to Theorem 4. The regularity conditions imposed on $g(x)$ there are trivially satisfied, since $g(x)=U(x) \xi U(x)^{-1}$, with $U(x)$ unitary and strongly continuous in $x$.

This completes the verification of the conditions of Theorem 4 and its complement. (4.2) now gives the convergence of $\lambda_{n}(z)$ to $\lambda$ as $n \rightarrow \infty$ in measure on any bounded interval of $(0, \infty)$.

REMARK. The conditions on the potential in Theorem 5 are quite strong, and it would be desirable to relax them. Possibly one could obtain a better result simply by a different choice of $U(z)$. However, it seems more likely that it would be necessary to prove a version of the approximation theorem (the second part of Theorem 4) requiring only smoothness but not analyticity of the family of operators $\{K(x)\}$. In our proofs the approximation theorem is linked closely to the representation theorem (4.1), which requires analyticity for its formulation. The lemma proved in Appendix A represents a step in this direction.

Perhaps more serious is the absence in Theorem 5 of any discussion of the rate of convergence of the approximations, and of the dependence of the convergence rate on the choice of trial functions.

A similar discussion could be given using Theorem 4 of the con- 
vergence of the Kohn approximations for the phase shifts for higher order partial waves. If the potential is no longer assumed central, the analogs of Nuttall's equations (5.5), (5.7) can still be set up, but it is not clear whether they would fall within the scope of Theorem 4 .

APPENDIX A. In this appendix we present a lemma (A3) which may be used in place of Lemma 11 in the proof of the approximation part of Theorem 3; the argument is a refinement of that used by Nuttall [14] in his proof of his convergence theorem for Kohn approximations (see $\S 5$ ). It is of interest because it may be of use in proving a version of approximation theorem not requiring analyticity for $K(x)$ (cf. final remark of $\S 5$ ).

As a consequence of the continuity properties of the Hilbert transform $\overparen{T(\sigma) \nu}$ we obtain

Lemma A1. The restriction of $T(\sigma) \nu$ to a compact set $J$ is continuous in $\sigma$ and in $\nu$.

Lemma A2. Let $\nu$ be a finite sum of positive masses, $\Delta$ and $F$ $\mu$-measurable subsets of $\boldsymbol{R}$, with compact closures. Then

$$
\mu\{x \in \Delta \mid \widetilde{\nu}(x) \in F\}=\int_{F} d \mu(\sigma)[T(\sigma) \nu](\Delta) .
$$

Proof. It suffices to prove (A. 1) for the case in which $F$ and $\Delta$ are intervals. Let $\tilde{\nu}(x)=\sum\left(c_{i} / x-a_{i}\right)$. On each interval $\left(a_{i}, a_{i+1}\right)$, $\tilde{\nu}(x)$ is strictly decreasing, and so has an inverse $\rho_{i}(\sigma)$

$$
\mu\{x \in \Delta \mid \tilde{\nu}(x) \in F\}=-\int_{F} d \mu(\sigma) \sum_{i}^{\prime} \frac{d \rho_{i}}{d \sigma}
$$

(the prime indicating that the summation is over $i$ such that $\rho_{i}(\sigma) \in \Delta$ ). But

$$
\begin{aligned}
-\frac{d \rho_{i}}{d \sigma} & =-\left.\left(\frac{d \tilde{\nu}}{d x}\right)^{-1}\right|_{x=\rho_{i}(\sigma)} \\
& =\text { residue of }(\sigma-\tilde{\nu}(z))^{-1} \text { at } z=\rho_{i}(\sigma)
\end{aligned}
$$

so

$$
-\sum_{i}^{\prime} \frac{d \rho_{i}}{d \sigma} i=[T(\sigma) \nu](\Delta)
$$

Note that the estimate given in [20] (Theorem 8) for the distribution function of the Hilbert transform of the restriction of Lebesgue measure $\mu$ to a set of finite $\mu$ measure, shows that (A.1) is not true for all positive measures $\nu$.

Lemma A3. Let $\left\{\boldsymbol{\nu}_{N}\right\}$ be a bounded sequence of positive masses on 
$\boldsymbol{R}$, each a finite sum of point masses. Suppose that, for each $N$, the support of $\nu_{N}$ is contained in a finite interval I (independent of $N$ ). Let $g(x)$ be a real-ralued $\mu$ measurable function on $\boldsymbol{R}, J$ a $\mu$ measurable subset of $\boldsymbol{R}$ with compact closure. Then, either some subsequence of $\left\{\nu_{N}\right\}$ converges to a positive measure $\nu$, such that, on some subset of $J$ of positive $\mu$ measure, both (a) and (b) hold

$$
\begin{aligned}
\tilde{\nu}(x)+g(x) & =0 \\
(d \nu / d \mu)(x) & =0
\end{aligned}
$$

or the sequence $\varphi_{N}(x)=\left(\tilde{\nu}_{N}(x)+g(x)\right)^{-1}$ is bounded in measure $(\mu)$ on $J$.

Proof. As in Lemma 11, a compactness argument shows that it suffices to consider the case in which the sequence $\nu_{N}$ is convergent to $\nu$ (say). If $\nu$ is the zero measure, (b) holds for all $x$ and, since $\left\|\boldsymbol{\nu}_{N}\right\| \rightarrow 0$ as $N \rightarrow \infty$, Lemma 5 implies $\widetilde{\nu}_{N}(x) \rightarrow 0$ in measure $(\mu)$. But then it is clear that $\left\{\varphi_{N}(x)\right\}$ is bounded in measure on $J$ unless $g(x)=0$ on a subset of $J$ of positive measure, which is the assertion of the lemma. Suppose then that $\nu$ is not the zero measure.

Suppose (a), (b) do not hold on a subset of $J$ of positive measure. To prove that $\left\{\varphi_{N}(x)\right\}$ is bounded in measure on $J$, we must show that for every $\eta>0$ we have, for sufficiently small $\varepsilon$ and large $N$,

$$
\mu\left\{x \in J|| \tilde{\nu}_{N}(x)+g(x) \mid \leqslant \varepsilon\right\} \leqslant \eta .
$$

By Lusin's theorem we may find a continuous function $g_{y}(x)$ and a compact subset $J_{\eta}$ of $J$ such that

$$
\mu\left(J-J_{\eta}\right) \leqslant \eta / 2 \quad \text { and } \quad g(x)=g_{\eta}(x) \quad x \in J_{\eta}
$$

and it then suffices to show (for sufficiently large $N$, and small $\varepsilon$ )

$$
\mu\left\{x \in J_{r_{r}}|| \widetilde{\nu}_{N}(x)+g_{r}(x) \mid \leqslant \varepsilon\right\} \leqslant \eta / 2 .
$$

Thus we may suppose $g(x)$ continuous, and $J$ compact. By supposition

$$
|(\widetilde{\nu}(x)+g(x))+i \pi(d \nu / d \mu)(x)| \neq 0 \text { a.e. }(\mu) \text { on } J
$$

so (again by deleting a set of arbitrarily small $\mu$ measure) we can suppose

$$
|(\widetilde{\nu}(x)+g(x))+i \pi(d \nu / d \mu)(x)| \geqslant c>0 \text { on } J .
$$

Consider the measures $T(\sigma) \nu$ defined following Lemma 12. Denote by $S$ the union of the singular supports of $T(\sigma) \nu$ ( $\sigma$ rational). Then $S$ is of $\mu$ measure zero, and if $B$ is any relatively compact Borel subset of $R$ of $\mu$ measure zero, such that $B \cap S=\varnothing$, and $\sigma$ a real 
number, we may choose a sequence $\sigma_{j}$ of rationals converging to $\sigma$, and obtain

$$
\begin{aligned}
{[T(\sigma) \nu](B) } & =\lim _{j \rightarrow \infty}\left[T\left(\sigma_{j}\right) \nu\right](B) \text { by Lemma } \mathrm{A} 1 \\
& =0 \quad \text { by the definition of } S .
\end{aligned}
$$

Thus $S$ contains the singular support of $T(\sigma) \nu$ for any real $\sigma$. By a theorem proved in [15] (p. 139), $\widetilde{\nu}(z)$ has a.e. $(\mu)$ the nontangential boundary value

$$
\widetilde{\nu}(x)+i \pi(d \nu / d \mu)(x)
$$

(from the upper half-plane). We add to $S$ the points at which the boundary value does not exist. Then for $x \notin S(d T(\sigma) \nu / d \mu)(x)$ exists, and $(-\tilde{\mathcal{L}}(z)+\sigma)^{-1}$ has the nontangential boundary value

$$
\{(-\widetilde{\nu}(x)+\sigma)+i \pi(d \nu / d \mu)(x)\}^{-1} .
$$

Consider the restriction $\omega$ of $T(\sigma) \nu$ to a compact neighborhood of $x$. According to the definition of $T(\sigma) \nu$ the Hilbert transform $\tilde{\omega}(z)$ differs from $(-\widetilde{\mathcal{\nu}}(z)+\sigma)^{-1}$ by a function holomorphic in the neighborhood of $x$; this function will take real values on the real axis. The nontangential boundary value of $\tilde{\omega}(z)$ at $x$ therefore exists, and its imaginary part is equal to the imaginary part of (A.3). Since $(d \omega / d \mu)(x)=(d T(\sigma) \nu / d \mu)(x)$ exists, it follows by another theorem in [15] (p. 34) that

$$
\text { (A.4) } \quad \frac{d T(\sigma)}{d \mu} \nu(x)=\frac{d \nu}{d \mu}(x)\left|(-\widetilde{\nu}(x)+\sigma)^{-1}+i \pi \frac{d \nu}{d \mu}(x)\right|^{-2} \text {. }
$$

By deleting from $J$ a set of arbitrarily small $\mu$ measure we can suppose $J \cap S=\varnothing$.

Given $\varepsilon>0$, we can partition $J$ into a finite number of $\mu$-measurable sets $\Delta_{i}$ on each of which $g$ has oscillation $\leqslant \varepsilon$. Let $g_{i}$ be a value of $g$ on $\Delta_{i}$. Then

$$
\text { (A.5) } \mu\left\{x \in J|| \widetilde{\nu}_{N}(x)+g(x) \mid \leqslant \varepsilon\right\} \leqslant \sum_{i} \mu\left\{x \in \Delta_{i}|| \widetilde{\nu}_{N}(x)+g_{i} \mid \leqslant 2 \varepsilon\right\} .
$$

By Lemma A2 the right side of (A.5) may be written

$$
\sum_{i} \int_{-g_{i}-2 \varepsilon}^{-g_{i}+2 \varepsilon} d \mu(\sigma)\left[T(\sigma) \nu_{N}\right]\left(\Delta_{i}\right) \text {. }
$$

Lemma A1 shows that, as $N \rightarrow \infty$. (A.6) has the limit

$$
\sum_{i} \int_{-g_{i}-2 \varepsilon}^{-g_{i}+2 \varepsilon} d \mu(\sigma)[T(\sigma) \nu]\left(\Delta_{i}\right) \text {. }
$$

(The convergence of $\left[T(\sigma) \nu_{N}\right]\left(\Delta_{i}\right)$ to $[T(\sigma) \nu]\left(\Delta_{i}\right)$ can be shown to be bounded in $\sigma$.$) Since \Delta_{i} \cap S=\varnothing$ 


$$
[T(\sigma) \nu]\left(\Delta_{i}\right)=\int_{\Delta_{i}} d \mu(s) \frac{d T(\sigma) \nu(s)}{d \mu} .
$$

For $s \in \Delta_{i}$ and $\left|\sigma+g_{i}\right| \leqslant 2 \varepsilon,|\sigma+g(s)| \leqslant 3 \varepsilon$ so, if we choose $\varepsilon \leqslant c / 6$, (A.2) gives

$$
|(-\widetilde{\nu}(s)+\sigma)+i \pi(d \nu / d \mu)(s)| \geqslant c / 2>0
$$

and from (A.4) and (A.8) we obtain

$$
[T(\sigma) \nu]\left(\Delta_{i}\right) \leqslant 4 c^{-2} \nu\left(\Delta_{i}\right) .
$$

This gives for (A.7) the bound

$$
\sum_{i} 4 \varepsilon \cdot 4 c^{-2} \nu\left(\Delta_{i}\right)=16 c^{-2} \nu(J) \varepsilon
$$

This bound $\rightarrow 0$ as $\varepsilon \rightarrow 0$.

Lemma A3 implies, in particular, that if $d \nu / d \mu \neq 0$ a.e. $(\mu)$ on $J$, then no subsequence of $\widetilde{\nu}_{N}(x)$ converges on any subset of $J$ of positive $\mu$ measure. Convergence to $+\infty$ or $-\infty$ on a subset of $J$ of positive $\mu$ measure is also excluded since $\left\{\tilde{\nu}_{N}(x)\right\}$ is bounded in measure by Lemma 5. This negative result is to be contrasted with the positive result proved in [20] that norm convergence of a sequence $\left\{\boldsymbol{\nu}_{N}\right\}$ of measures implies convergence in measure for the sequence $\left\{\widetilde{\nu}_{N}(x)\right\}$.

An alternative proof of Lemma 11 (not relying on Lemma 10) may be obtained by combining Lemma A3 with the theorem of Lusin and Privalov [15] (p. 212) according to which a holomorphic function in $\operatorname{Im} z>0$ (here $\tilde{\nu}(z)+g(z)$ ), which has nontangential boundary value zero on a set of positive $\mu$ measure, vanishes identically.

ACKNowledgments. The author thanks Professor J. Nuttall for suggesting the problem, and for valuable discussions. He thanks Texas A \& M University for its hospitality, while the work was begun, and his colleagues Professors F. Brownell, E. L. Stout, and M. Hackman for helpful remarks.

\section{REFERENCES}

1. J. Aguilar and J. M. Combes, A class of analytic perturbations for one-body Schroedinger Hamiltonians, Commun. Math. Phys., 22 (1971), 269-279.

2. N. K. Bary, Treatise on Trignometric Series II, MacMillan, New York, 1964.

3. F. H. Brownell, Some integral limits associated with Hilbert transforms, J. Math. Anal. Appl., 12 (1965), 401-409.

4. - Some integral limits associated with Hilbert transforms II, J. Math. Anal. Appl., 16 (1966), 15-30.

5. N. Dunford and J. T. Schwartz, Linear Operators, Part II, Wiley, New York, 1963.

6. —, Linear Operators, Part III: Spectral Operators, Wiley, New York, 1971. 
7. C. R. Garibotti, The Schwinger variational Principle and Padé approximants, Ann. Phys., 71 (1972), 486-496.

8. M. L. Goldberger and K. M. Watson, Collision Theory, Wiley, New York, 1964.

9. T. Kato, Perturbation Theory for Linear Operators, Springer-Verlag, New York, 1966.

10. M. G. Krein and H. Langer, On the spectral function of a self-adjoint operator in a space with indefinite metric, Dokl. Akad. Nauk 152, (1963), 39-42.

11. H. Langer, Lecture Notes on J-Self-adjoint Operators, University of Toronto, 1967. 12. - Spectralfunktionen einer Klasse J-selbstadjungierten Operatoren, Mathematische Nachrichten, 33 (1967), 107-120.

13. G. D. McCartor, Topics in Scattering Theory, Ph.D. Thesis, Texas A \& M University, 1969.

14. J. Nuttall, The convergence of the Kohn variational method, Annals of Physics, 10 (1969), 428-443.

15. I. I. Privalov, Randeigenschaften Analytischer Funktionen, VEB, Deutscher Verlag der Wissenschaften, Berlin, 1956.

16. C. R. Putnam, Commutation Properties of Hilbert Space Operators and Related Topics, Springer-Verlag, Now York, 1967.

17. B. Simon, Quantum Mechanics for Hamiltonians Defined as Quadratic Forms, Princeton University Press, 1971.

18. M. H. Stone, Linear transformations in Hilbert space, Amer. Math. Soc., 1932.

19. R. F. Streater and A. S. Wightman, PCT, Spin, and Statistics, and All That, W. A. Benjamin, New York, 1964.

20. A. Zygmund, Intégrales Singulières, Springer Notes 204, 1971.

Received May 25, 1972 and in revised form August 24, 1972. Work supported in part by the U.S. Air Force Office of Scientific research, Office of Aerospace Research, under Grant No. 71-1979.

UNIVERSITY OF WASHINGTON 


\section{PACIFIC JOURNAL OF MATHEMATICS}

\section{EDITORS}

D. Gilbarg AND J. MILGRAM

Stanford University

Stanford, California 94305

\section{R. A. Beaumont}

University of Washington

Seattle, Washington 98105

\section{J. DUGUNDJI*}

Department of Mathematics University of Southern California Los Angeles, California 90007

RICHARD ARENS

University of California Los Angeles, California 90024

\section{ASSOCIATE EDITORS}
E. F. BECKENBACH
B. H. NEUMANN
F. WOLF
K. YoshidA

\section{SUPPORTING INSTITUTIONS}

\author{
UNIVERSITY OF BRITISH COLUMBIA \\ CALIFORNIA INSTITUTE OF TECHNOLOGY \\ UNIVERSITY OF CALIFORNIA \\ MONTANA STATE UNIVERSITY \\ UNIVERSITY OF NEVADA \\ NEW MEXICO STATE UNIVERSITY \\ OREGON STATE UNIVERSITY \\ UNIVERSITY OF OREGON \\ OSAKA UNIVERSITY
}

\author{
UNIVERSITY OF SOUTHERN CALIFORNIA \\ STANFORD UNIVERSITY \\ UNIVERSITY OF TOKYO \\ UNIVERSITY OF UTAH \\ WASHINGTON STATE UNIVERSITY \\ UNIVERSITY OF WASHINGTON \\ AMERICAN MATHEMATICAL SOCIETY \\ NAVAL WEAPONS CENTER
}

The Supporting Institutions listed above contribute to the cost of publication of this Journal, but they are not owners or publishers and have no responsibility for its content or policies.

Mathematical papers intended for publication in the Pacific Journal of Mathematics should be in typed form or offset-reproduced, (not dittoed), double spaced with large margins. Underline Greek letters in red, German in green, and script in blue. The first paragraph or two must be capable of being used separately as a synopsis of the entire paper. Items of the bibliography should not be cited there unless absolutely necessary, in which case they must be identified by author and Journal, rather than by item number. Manuscripts, in duplicate if possible, may be sent to any one of the four editors. Please classify according to the scheme of Math. Rev. Index to Vol. 39. All other communications to the editors should be addressed to the managing editor, Richard Arens, University of California, Los Angeles, California, 90024.

50 reprints are provided free for each article; additional copies may be obtained at cost in multiples of 50 .

The Pacific Journal of Mathematics is issued monthly as of January 1966. Regular subscription rate: $\$ 48.00$ a year (6 Vols., 12 issues). Special rate: $\$ 24.00$ a year to individual members of supporting institutions.

Subscriptions, orders for back numbers, and changes of address should be sent to Pacific Journal of Mathematics, 103 Highland Boulevard, Berkeley, California, 94708.

PUBLISHED BY PACIFIC JOURNAL OF MATHEMATICS, A NON-PROFIT CORPORATION

Printed at Kokusai Bunken Insatsusha (International Academic Printing Co., Ltd.), 270, 3-chome Totsuka-cho, Shinjuku-ku, Tokyo 160, Japan.

* C. DePrima will replace J. Dugundji until August 1974.

Copyright (C) 1973 by

Pacific Journal of Mathematics

All Rights Reserved 


\section{Pacific Journal of Mathematics}

\section{Vol. 47, No. $2 \quad$ February, 1973}

David Parham Bellamy, Composants of Hausdorff indecomposable continua; a mapping approach ........................ 303

Colin Bennett, A Hausdorff-Young theorem for rearrangement-invariant spaces ...........................................

Roger Daniel Bleier and Paul F. Conrad, The lattice of closed ideals and $a^{*}$-extensions of an abelian l-group ...

Ronald Elroy Bruck, Jr., Nonexpansive projections on subsets of Banach

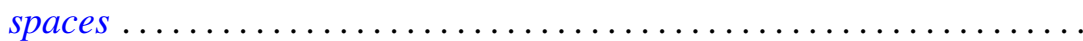

Robert C. Busby, Centralizers of twisted group algebras ............. 357

M. J. Canfell, Dimension theory in zero-set spaces ................ 393

John Dauns, One sided prime ideals ........................ 401

Charles F. Dunkl, Structure hypergroups for measure algebras . . . . . . . . . 413

Ronald Francis Gariepy, Geometric properties of Sobolev mappings ...... 427

Ralph Allen Gellar and Lavon Barry Page, A new look at some familiar spaces of intertwining operators ...........................

Dennis Michael Girard, The behavior of the norm of an automorphism of the

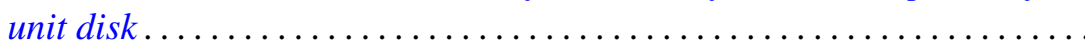

George Rudolph Gordh, Jr., Terminal subcontinua of hereditarily

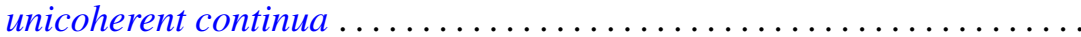

Joe Alston Guthrie, Mapping spaces and cs-networks. .

Neil Hindman, The product of $F$-spaces with $P$-spaces . 473

M. A. Labbé and John Wolfe, Isomorphic classes of the spaces $C_{\sigma}(S)$

Ernest A. Michael, On k-spaces, $k_{R}$-spaces and $k(X) \ldots$

Donald Steven Passman, Primitive group rings .

C. P. L. Rhodes, A note on primary decompositions of a pseudovaluation ...

Muril Lynn Robertson, A class of generalized functional differential equations

Ruth Silverman, Decomposition of plane convex sets. $I$.

Ernest Lester Stitzinger, On saturated formations of solvable Lie algebras................................

B. Andreas Troesch, Sloshing frequencies in a half-space by Kelvin inversion ...

L. E. Ward, Fixed point sets .

Michael John Westwater, Hilbert transforms, and a problem in scattering

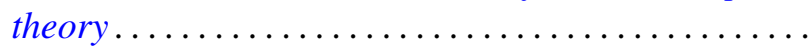

Misha Zafran, On the spectra of multipliers... 